SVU- International Journal of Veterinary Sciences, 3 (1): 100-122, 2020.

Print ISSN: 2535-1826

Online ISSN: 2535-1877

\title{
Molecular detection of antibiotic resistance and virulence genes in staphylococcus species isolated from human and poultry
}

\author{
M.S. Ahmed ${ }^{1 *}$, M.W. ABD AL-AZEEM ${ }^{2}$, Hams M.A. Mohamed ${ }^{2}$ \\ ${ }^{1 *}$ Microbiology lab of Assiut University Hospital, Egypt. ${ }^{2}$ Department of Microbiology, Faculty of \\ Veterinary Medicine, South Valley University, Qena, Egypt.
}

\section{Abstract}

Staphylococcus species are important potential pathogens that can give rise to acute and chronic diseases for poultry and humans. Since they carry virulence and antibiotic resistance genes which can be transmitted between food producing animals and humans during various modes, the major one being food chain and cause health hazards for the consumers. Therefore, the current study was intended to inspect the incidence rate of Staphylococcus species isolated from chicken and patients, detection the antimicrobial susceptibility of these isolates by using Vitek2 system and affirmation the attendance of genes coding for pathogenicity and antimicrobial resistance in detected isolates by PCR. To achieve these 200 samples were collected from poultry farms and patient (100 for each) in disparate districts in Assiut province, to be subjected to bacteriological examination. The results exposed that the incidence of Staphylococcus spp. was $35 \%$ and $45 \%$ in poultry and human samples respectively on mannitol agar. Vitek2 system differentiated the poultry isolates to 11 isolates as coagulase-positive Staphylococci (CoPS) and 24 isolates as coagulase-negative Staphylococci (CoNS). While 20 human isolates identified as CoPS and 25 isolates consigned as CoNS. Antibiogram refined that $45.7 \%$ and $53.3 \%$ of Staphylococcus isolates from poultry and human were identified as methicillin-resistant Staphylococci respectively, also Staphylococcal spp. clarified a resistance to different types of antimicrobials such as penicillin, tetracycline clindamycin. Vitek2 system showed a inordinate ability to differentiate Staphylococcus species and evaluate its antimicrobial susceptibility which was convoluted by conventional method. PCR results revealed that the Staphylococcus isolated from poultry and human were harbored genes encoding for pathogenicity (coa, hld and $p v l$ ) and antimicrobial resistance (mecA, vanA, cfr and blaZ) while none of the isolates harbored sei and seh genes. The phylogenetic analysis constructed on 16SrRNA sequencing of Staphylococcus spp. showed a relationship between these species isolated from poultry and human. So, the obtained results emphasized the importance of reducing the unwarranted use of antimicrobial agents and implementation of sanitary hygienic procedures in poultry production.

Keywords: Staphylococcus species, Vitek2 system, Virulence genes, antimicrobials resistance genes, $16 S r R N A$ sequencing, poultry, human

DOI: 10.21608/svu.2020.25542.1045 Received: March 9, 2020 Accepted: April 23, 2020 Published: April 30, 2020. *Corresponding Author: M. S. Ahmed. E-mail: vetmohs.salah@yahoo.com

Citation: Ahmed et al., 2020, Molecular detection of antibiotic resistance and virulence genes in staphylococcus species isolated from human and poultry. SVU-IJVS 2020, 3(1): 100-122.

Copyright: () Salah et al. This is an open access article distributed under the terms of the creative common attribution license, which permits unrestricted use, distribution and reproduction in any medium provided the original author and source are created.

Competing interest: The authors have declared that no competing interest exists. 


\section{INTRODUCTION}

Staphylococcus are widely distributed in water, soil and air, in addition to its isolation from various animal species, including poultry and its genus is present in 70 species (Götz et al., 2006).Poultry meat is the most important source of human food poisoning (Kadariya et al., 2014).Staphylococcal food-borne disease induced by poultry meat became an evident problem reflected negatively on the industry of poultry, causing drawbacks on public health and making difficulty for the medical and veterinary organization (Teramoto et al., 2016).

Staphylococcus species, generally dubbed as coagulase-negative-Staphylococci (CoNS) and acquired its value as they have been responsible for multiple infections in humans and animals (Vuong and Otto, 2002).

Staph.aureus is the most critical species within this genus, recognized as coagulasepositive Staphylococcus(CoPS) as well as, be one of the causes of food intoxication (Cunha, 2009). This microorganism is the etiological factor causing several avian diseases such as arthritis, septicemia, omphalitis,..etc (Smyth and McNamee, 2001). These Staphylococcal infectious diseases of chickens are an economic threat and they are viewed as worldwide burden (Lowder et al., 2009).

CoNS are causing nosocomial infections in neonatal intensive care units and uncommon food poisoning bacteria (Tong et al., 2015).Various researches on CoNS and more than 15,000 references, reflecting the increasing of medical influence of these bacteria (Becker et al., 2014)due to the possible spreading of antimicrobial resistant bacteria and antimicrobial resistant genes (ChajęckaWierzchowska et al., 2015), although, CoNS is documented as a very useful bacteria in the technology and hygiene of food production and preservation (Šušković et al., 2010).

The virulence genes are communally responsible for the pathogenicity of this organism, like Staphylococcal protein A (spa), coagulase protein (coa), Staphylococcal enterotoxins A to E collagen adhesion gene (cna), toxic shock syndrome toxin 1 (tst), exfoliative toxins $($ eta $a t b)$, and leucocidins (Pereira et $a \boldsymbol{l}$., 2009).

The appreciation of antimicrobial resistance in food borne pathogens inventing from food producing animals such as chicken that has been recorded in developing countries (Van et al., 2007).The proclivity for Staphylococci to develop antimicrobial resistance is a reason for great worry in both human and animals (Vanderhaeghen $\boldsymbol{e t}$ al., 2010).

The undesirable antimicrobial resistance has mostly been risked as result of unreasonable use of antibiotics in producing animals (Adesiji et al., 2014).Amongst all types of resistance, methicillin resistant Staph.aureus (MRSA) is considered as critical, as it had been confirmed as the source of acquired infections associated with high rate of bacterial mortality worldwide (Tiemersma et al., 2004).

Automated systems VITEK 2 analyses have proven to be an accurate technique to differentiate Staphylococcal species (Sukru et al., 2018). It is used for the identification of isolated colonies to the genus and species levels (Jackson et al., 2013). Rapid and accurate methods for identification of food borne pathogens are important for microbiological safety. In previous recent years, polymerase chain reaction (PCR) was proven as the most suitable method for fast, sensitive and unrestricted detection of pathogenic bacteria in food (Kim and Kim, 2017). 
The aim of the present study was isolation and identification the Staphylococcus species isolated from poultry and human samples by using VITEK2 system, also detection of genes encoding for pathogenicity (coa,hld,sei,pvl and seh) and antimicrobial resistance genes (mecA,cfr,van $\mathrm{A}$ and blaZ) in Staphylococcus isolates by PCR and evaluation the relationship between isolated species from poultry and humans by Phylogenesis of the sequenced isolates.

\section{MATERIALS AND METHODS}

\section{Ethical approval}

The study was carried out in line with all applicable regulations and legislation of Commission for Ethics in Scientific Research, faculty of medicine, Assiut University, Egypt. The date of ethical approval of the research was $1 / 1 / 2018$.

\section{Samples collection}

A total of 200 samples were collected aseptically from poultry farms and university hospital in Assiut province (100 for each) during period between March to September 2018. Poultry samples included liver, tarsal joint and intestine were removed from each bird according to Monecke etal. (2013).Also patient swabs (Abscess, conjunctivitis, otitis, and urine) were gathered according Strommenger etal. (2008).

\section{Preparation of samples}

\section{Poultry samples:}

Slices of liver, tarsal joints and were immersed in test tubes containing nutrient broth for overnight (Mkize, 2016) and nearly one gram of intestinal content was putted in a centrifuge tube containing $9 \mathrm{ml}$ of sterile phosphate buffered saline (PBS) $\mathrm{pH}$ 7.4, and mixed by vortex with glass beads ( $4 \mathrm{~mm}$ in diameter) for 3 minutes. Debris was expelled by centrifugation at 700xg for 1 minute (Seidavi et al., 2010). One milliliter of supernatant was inoculated in a tube contained $9 \mathrm{ml}$ Brain
Heart Infusion broth (BHI) and incubated at $37^{\circ} \mathrm{C}$ for $24 \mathrm{~h}$

\section{Patient samples:}

The patient swabs were immersed in a tube contained $9 \mathrm{ml}$ Brain Heart Infusion broth (BHI) and incubated at $37^{\circ} \mathrm{C}$ for $24 \mathrm{~h}$.

Isolation and identification of
Staphylococcus species

Aloopful of BHI broth was streaked on mannitol salt agar at $37^{\circ} \mathrm{C}$ for $24 \mathrm{~h}$ (Gharajalar and Shahbazi, 2018). The obtained colonies were plated onto Sheep blood agar , Baird-Parker agar for identification of Staph. aureus from other species (Son et al., 2010) and Oxacillin Resistance Screening Agar Base(ORSAB) agar for identification of methicillinresistant Staphylococcus isolates of poultry and human(Nahimana et al., 2006).

Morphological examination of the suspected colonies was done by using Gram staining and biochemical identification of isolates on the base of catalase activity, coagulase (rabbit plasma) and oxidase test (Fijalkowski et al., 2016). Identification of Staphylococcus species and antibiotic susceptibility using VITEK 2 compact ${ }^{\circledR}$

The identification of Staphylococcus spp. was done by VITEK GP (Gram Positive)on all obtained isolates from mannitol salt agar according to the cards with reference number 21342, also antimicrobials susceptibilities were done conferring to antibiogram cards AST-GP 67 with reference number 22226 (Sukru et al., 2018).

Detection of virulence and antimicrobial resistance genes in Staphylococcal species by PCR

DNA was extracted from ten Staphylococcal isolates belonging to different species according to Mansour et al., (2017), after refreshment of these isolates in $5 \mathrm{ml}$ of $\mathrm{BHI}$ broth at $35^{\circ} \mathrm{C}$ $\left( \pm 2^{\circ} \mathrm{C}\right)$ for $24 \mathrm{hrs}$ by using QIAamp DNA 
Mini kit (Qiagen, Germany, GmbH) on bases of manufacturer's instruction.

Different primers were used as $16 \operatorname{Sr} R N A$ primer derived from Staphylocoocus genome for confirmation our isolates, in addition to (coa, sei, seh, hld and $p v l$ ) for detection virulence and $(m e c \mathrm{~A}, v a n \mathrm{~A}, c f r$ and blaZ) for antimicrobial resistance (Table 1). Uniplex PCR amplification reaction was performed in a final volume of $25 \mu 1$, composed of $6 \mu 1$ DNA, $12.5 \mu 1$ Mastermix(Emerald Amp GT PCR), $1 \mu 1$ for each primer and $4.5 \mu \mathrm{l}$ of PCR grade water. Reactions were implemented in thermal cycler (MJ Research, Inc. Watertown, MA) with the following program: initial denaturation at $95^{\circ} \mathrm{C}$ for 5 min followed by 45 cycles of $95^{\circ} \mathrm{C}$ for $45 \mathrm{sec}, 50^{\circ} \mathrm{C}$ for $45 \mathrm{sec}$ and $72^{\circ} \mathrm{C}$ for $1 \mathrm{~min}$ with a final extension at $72^{\circ} \mathrm{C}$ for $10 \mathrm{~min}$. The amplicons size (bp) were detected by electrophoresis on $1.5 \%$ agarose gel (BioshopR, Candainc.) stained with ethidium bromide, then visualized in a UV transilluminator.

Amplification and sequencing of universal $16 \mathrm{~S} r \mathrm{RNA}$ gene

The amplification of universal $16 S \mathrm{rRNA}$ gene was done in 5 isolates of Staph. aureus ( 2 from poultry and 3 from human) and 2 isolates of Staph. lentus ( one isolates from each poultry and human) by using the universal primers $27 \mathrm{~F}$ (5'AGAGTTTGATCCTGGCTCAG-3') And 1492R

TACGGTTACCTTGTTACGACTT-3') at 1,500-bp (Liu et al.,2009). The reaction mixture was combined of $1 \mu \mathrm{l}$ of bacterial DNA, $1 \mu \mathrm{l}$ each primer 12.5 PCR master mix (Emerald Amp Max PCR Master) the mixture was completed by PCR water in a final reaction volume of $25 \mu \mathrm{l}$ after that, the program was run in thermal cycler (MJ Research, Inc. Watertown, MA). as follows: 30 cycles were done in a thermocycler; denaturation $95^{\circ} \mathrm{C}$ for $1 \mathrm{~min}$, annealing $54{ }^{\circ} \mathrm{C}$ for $1 \mathrm{~min}$, extension $72^{\circ} \mathrm{C}$ for $3 \mathrm{~min}$ and final extension time of $72{ }^{\circ} \mathrm{C}$ for $5 \mathrm{~min}$ (Alfatih et al.,2018). Amplified products were analyzed by product detected by electrophoresis on $1.5 \%$ agarose gel then visualized in a UV transilluminator. PCR products were purified using QIA quick PCR Product purification kit (Qiagen, Valencia, CA). For the sequencing reaction, a prism Big Dye terminator V3.1 Kit (applied Bio system) on DNA automated sequencer (applied Bio systems) were analyzed the sequence of $16 \mathrm{~S} r R N A$ gene in both directions forward and reverse. The sequence results of our isolates were analyzed by blasting on gene bank

https://blast.ncbi.nlm.nih.gov/Blast.cgi?PR OGRAM $=\quad$ blastn\&PAGE_TYPE $=$ BlastSearch\& LINK LOC=blasthome. Sequence alignment was done by multiple alignment algorithms in megalign (DNASTAR, Window version 3.12e).

\section{Phylogenetic analysis}

Phylogenetic tree was based on $16 S$ rRNA gene nucleotides sequence which was performed on our five Staph.aureus isolates (2isolates from poultry and 3isolates from human) and two Staph. lentus isolates (one for each) to inspect the identity of amplified fragment of $16 \mathrm{~S}$ rRNA gene in our isolates with Staphylococcus isolates reference strains registered with Gene bank using MEGA version2.1 (Kumar et al.,2001).

\section{Statistical analysis}

Statistics were done using IBM SPSS19.0. The person chi-squar test was used to inspect the relation between variables Statistically significant was defined as $\mathrm{P}<0.05$ 
Ahmed et al., 2020

SVU-IJVS, 3 (1): 100-122

Table (1): Nucleotidesequences, operative protocols and amplicons size (bp) of primers

\begin{tabular}{|c|c|c|c|c|}
\hline Gene & Oligonucleotide sequences $(5-3)$ & $\begin{array}{l}\text { Amplion } \\
\text { size (bp) }\end{array}$ & PCR conditions & References \\
\hline $16 \mathrm{r} R N A$ & $\begin{array}{l}\text { F: AAC TCT GTT ATT AGG GAA GAA CA } \\
\text { R: CCA CCT TCC TCC GGT TTG TCA CC }\end{array}$ & 250 & $\begin{array}{l}\text { Initial denaturation: } 95{ }^{\circ} \mathrm{C} \text { for } 5 \mathrm{~min} \\
\text { Amplification }(45 \text { cycles of }) \\
\text { Denaturation: } 95^{\circ} \mathrm{C} \text { for } 45 \mathrm{~s} \\
\text { Annealing: } 50^{\circ} \mathrm{C} \text { for } 45 \mathrm{~s} \\
\text { Extension: } 72^{\circ} \mathrm{C} \text { for } 1 \mathrm{~min} \\
\text { Final extension: } 72^{\circ} \mathrm{C} \text { for } 10 \mathrm{~min}\end{array}$ & (Zhang et al., 2004) \\
\hline $\operatorname{coa}$ & $\begin{array}{l}\text { F:ACCACAAGGTACTGAATAACG } \\
\text { R:TGCTTTCGATTGTTCGATGC }\end{array}$ & 987 & $\begin{array}{l}\text { Initial denaturation: } 95^{\circ} \mathrm{C} \text { for } 5 \mathrm{~min} \\
\text { Amplification }(45 \text { cycles of }) \\
\text { Denaturation: } 95^{\circ} \mathrm{C} \text { for } 45 \mathrm{~s} \\
\text { Annealing: } 55^{\circ} \mathrm{C} \text { for } 45 \mathrm{~s} \\
\text { Extension: } 72^{\circ} \mathrm{C} \text { for } 1 \mathrm{~min} \\
\text { Final extension: } 72^{\circ} \mathrm{C} \text { for } 10 \mathrm{~min}\end{array}$ & (Veras et al., 2008) \\
\hline$s e i$ & $\begin{array}{l}\text { F:CAACTCGAATTTTCAACAGGTACC } \\
\text { R:CAGGCAGTCCATCTCCATCTCCTG }\end{array}$ & 466 & $\begin{array}{l}\text { Initial denaturation: } 95^{\circ} \mathrm{C} \text { for } 5 \mathrm{~min} \\
\text { Amplification }(45 \text { cycles of }) \\
\text { Denaturation: } 95^{\circ} \mathrm{C} \text { for } 45 \mathrm{~s} \\
\text { Annealing: } 50^{\circ} \mathrm{C} \text { for } 45 \mathrm{~s} \\
\text { Extension: } 72^{\circ} \mathrm{C} \text { for } 1 \mathrm{~min} \\
\text { Final extension: } 72^{\circ} \mathrm{C} \text { for } 10 \mathrm{~min}\end{array}$ & (Pereira et al., 2009) \\
\hline seh & $\begin{array}{l}\text { R: CAA CTG CTG ATT TAG CTC AG } \\
\text { F:GTC GAA TGA GTA ATC TCT AGG }\end{array}$ & 360 & $\begin{array}{l}\text { Initial denaturation: } 95^{\circ} \mathrm{C} \text { for } 5 \mathrm{~min} \\
\text { Amplification }(45 \text { cycles of }) \\
\text { Denaturation: } 95^{\circ} \mathrm{C} \text { for } 45 \mathrm{~s} \\
\text { Annealing: } 50{ }^{\circ} \mathrm{C} \text { for } 45 \mathrm{~s} \\
\text { Extension: } 72^{\circ} \mathrm{C} \text { for } 1 \mathrm{~min} \\
\text { Final extension: } 72^{\circ} \mathrm{C} \text { for } 10 \mathrm{~min}\end{array}$ & $\begin{array}{r}\text { (Monday and Bohach, } \\
1999)\end{array}$ \\
\hline hld & $\begin{array}{l}\text { F: } \\
\text { AAGAATTTTTATCTTAATTAAGGAAGGAGTG } \\
\text { R: TTAGTGAATTTGTTCACTGTGTCGA }\end{array}$ & 111 & $\begin{array}{l}\text { Initial denaturation: } 95^{\circ} \mathrm{C} \text { for } 5 \mathrm{~min} \\
\text { Amplification }(45 \text { cycles of }) \\
\text { Denaturation: } 95^{\circ} \mathrm{C} \text { for } 45 \mathrm{~s} \\
\text { Annealing: } 60^{\circ} \mathrm{C} \text { for } 45 \mathrm{~s} \\
\text { Extension: } 72^{\circ} \mathrm{C} \text { for } 1 \mathrm{~min} \\
\text { Final extension: } 72^{\circ} \mathrm{C} \text { for } 10 \mathrm{~min}\end{array}$ & (Jarraud et al., 2009) \\
\hline$p v l$ & $\begin{array}{l}\text { F:ATCATTAGGTAAAATGTCTGGACATGATCCA } \\
\text { R:GCATCAAGTGTATTGGATAGCAAAAGC }\end{array}$ & 433 & $\begin{array}{l}\text { Initial denaturation: } 95^{\circ} \mathrm{C} \text { for } 5 \mathrm{~min} \\
\text { Amplification }(45 \text { cycles of }) \\
\text { Denaturation: } 95^{\circ} \mathrm{C} \text { for } 45 \mathrm{~s} \\
\text { Annealing: } 50{ }^{\circ} \mathrm{C} \text { for } 45 \mathrm{~s} \\
\text { Extension: } 72{ }^{\circ} \mathrm{C} \text { for } 1 \mathrm{~min} \\
\text { Final extension: } 72^{\circ} \mathrm{C} \text { for } 10 \mathrm{~min}\end{array}$ & (McClure et al., 2006) \\
\hline $\operatorname{mec} \mathbf{A}$ & $\begin{array}{l}\text { F:GTAGAAATGACTGAACGTCCGATAA } \\
\text { R:CCAATTCCACATTGTTTCGGTCTAA }\end{array}$ & 480 & $\begin{array}{l}\text { Initial denaturation: } 95{ }^{\circ} \mathrm{C} \text { for } 5 \mathrm{~min} \\
\text { Amplification }(45 \text { cycles of }) \\
\text { Denaturation: } 95{ }^{\circ} \mathrm{C} \text { for } 45 \mathrm{~s} \\
\text { Annealing: } 52^{\circ} \mathrm{C} \text { for } 45 \mathrm{~s} \\
\text { Extension: } 72{ }^{\circ} \mathrm{C} \text { for } 1 \mathrm{~min} \\
\text { Final extension: } 72^{\circ} \mathrm{C} \text { for } 10 \mathrm{~min}\end{array}$ & (Spanu et al., 2004) \\
\hline $\operatorname{van} \mathrm{A}$ & $\begin{array}{l}\text { F: GCGCGGTCCACTTGTAGATA } \\
\text { R: TGAGCAACCCCCAAACAGTA }\end{array}$ & 314 & $\begin{array}{l}\text { Initial denaturation: } 95^{\circ} \mathrm{C} \text { for } 5 \mathrm{~min} \\
\text { Amplification }(45 \text { cycles of }) \\
\text { Denaturation: } 95^{\circ} \mathrm{C} \text { for } 45 \mathrm{~s} \\
\text { Annealing: } 54{ }^{\circ} \mathrm{C} \text { for } 45 \mathrm{~s} \\
\text { Extension: } 72^{\circ} \mathrm{C} \text { for } 1 \mathrm{~min} \\
\text { Final extension: } 72^{\circ} \mathrm{C} \text { for } 10 \mathrm{~min}\end{array}$ & (Nam et al., 2012) \\
\hline$c f r$ & $\begin{array}{l}\text { F:TGAAGTATAAAGCAGGTTGGGAGTCA } \\
\text { R:ACCATATAATTGACCACAAGCAGC }\end{array}$ & 746 & $\begin{array}{l}\text { Initial denaturation: } 95^{\circ} \mathrm{C} \text { for } 5 \mathrm{~min} \\
\text { Amplification }(45 \text { cycles of }) \\
\text { Denaturation: } 95^{\circ} \mathrm{C} \text { for } 45 \mathrm{~s} \\
\text { Annealing: } 60^{\circ} \mathrm{C} \text { for } 45 \mathrm{~s} \\
\text { Extension: } 72^{\circ} \mathrm{C} \text { for } 1 \mathrm{~min} \\
\text { Final extension: } 72^{\circ} \mathrm{C} \text { for } 10 \mathrm{~min}\end{array}$ & $\begin{array}{r}\text { (Kehrenberg and Schwarz, } \\
2006)\end{array}$ \\
\hline blaZ & $\begin{array}{l}\text { F ACTTCAACACCTGCTGCTTTC } \\
\text { R TGACCACTTTTATCAGCAACC }\end{array}$ & 173 & $\begin{array}{l}\text { Initial denaturation: } 95^{\circ} \mathrm{C} \text { for } 5 \mathrm{~min} \\
\text { Amplification }(45 \text { cycles of }) \\
\text { Denaturation: } 95^{\circ} \mathrm{C} \text { for } 45 \mathrm{~s} \\
\text { Annealing: } 55^{\circ} \mathrm{C} \text { for } 45 \mathrm{~s} \\
\text { Extension: } 72^{\circ} \mathrm{C} \text { for } 1 \mathrm{~min} \\
\text { Final extension: } 72^{\circ} \mathrm{C} \text { for } 10 \mathrm{~min}\end{array}$ & (Martineau et al., 2000) \\
\hline
\end{tabular}

\section{Results}

\section{Results of conventional method}

The data illustrated in table (2) showed the result of bacterial examination for 200 samples were taken from poultry and human samples (100 for each) on mannitol salt agar, as followed, Staphylococcus spp. isolated from $35 \%$ and $45 \%$ of poultry and human samples 
Ahmed et al., 2020

SVU-IJVS, 3 (1): 100-122

respectively.94\% of poultry isolates were isolates into $31.4 \%$ and $44.4 \%$ coagulase obtained from intestinal content and 6\% obtained from joints while $60 \%$ of human isolates obtained from urine samples and $40 \%$ from abscesses. On the other hand, tube coagulase test divided the poultry and human positive and $68.6 \%$ and $55.6 \%$ were coagulase negative respectively. While ORSAB agar detected methicillin-resistant Staphylococci in $37.14 \%(13 / 35)$ and $42.22 \%(19 / 45)$ from Staphylococcal isolates respectively.

Table (2): Incidence of Staphylococcus spp. isolated from poultry and human

\begin{tabular}{|c|c|c|c|c|c|}
\hline \multirow[t]{2}{*}{ Source } & \multirow{2}{*}{$\begin{array}{l}\text { Number of } \\
\text { examined } \\
\text { samples }\end{array}$} & \multirow{2}{*}{$\begin{array}{c}\text { Suspected } \\
\text { isolates } \\
\text { on mannitol agar }\end{array}$} & \multicolumn{2}{|c|}{ Coagulase test } & \multirow[b]{2}{*}{$\begin{array}{l}\text { ORSAB agar positive } \\
\text { Staphylococci }\end{array}$} \\
\hline & & & Positive & Negative & \\
\hline Poultry & 100 & $35(35 \%)$ & $11(31.4 \%)$ & $24(68.8 \%)$ & $13(37.14 \%)$ \\
\hline Human & 100 & $45(45 \%)$ & $20(44.4 \%)$ & $25(55.6 \%)$ & $19(42.22 \%)$ \\
\hline Total & 200 & $80(40 \%)$ & $31(38.75 \%)$ & $49(61.25 \%)$ & $32(40 \%)$ \\
\hline
\end{tabular}

\section{Result of VITEK 2 Compact}

By using VITEK 2 system, 35 poultry isolates could be differentiated into17 Staphylococcal species, the highly identified species were Staph.aureus $14.3 \%$ (5/35) followed by Staph. lentus $8.5 \%$ (3/35), whereas the least species were Staph. lugdunensis, Staph. simulans and Staph. capitis $(2.86 \%$ for each). On the other hand, from 45 human isolates could differentiate 19 Staphylococcal species, the main species were Staph. aureus $31.1 \%$ (14/45) followed by Staph. haemolyticus and Staph. cohnii (3/45 for each) (6.7\%) (Table3) Statistical analysis showed a significant positive correlation between poultry and human isolates at $(\mathrm{R}=0.66$ at $\mathrm{p}<0.05)$

\section{Antibiogram for VITEK 2 Compact}

Compact antibiogram device was used for detection the antimicrobial resistance. The result of antibiogram showed in table (4) reveled that antimicrobial resistance profile of the 35Staphylococcus isolates from poultry samples to different antibiotics was investigated; none of the isolates were completely sensitive to the 13 tested antibiotics. High percentage of resistance was observed in tetracycline 28 (80\%), clindamycin $26(74.3 \%)$, penicillin $22(62.8 \%)$ and erythromycin18 (51.4\%). While, low resistance was noticed to gentamicin $6(17 \%)$ and trimethoprim / sulfamethazole $7(20 \%)$ between tested antibiotics.

Table (3): Results of Staphylococcus identification by using Vitek system

\begin{tabular}{|c|c|c|c|c|c|}
\hline \multirow{2}{*}{ Staphylococcus Species } & \multirow{2}{*}{ Source } & \multirow{2}{*}{ Number } & \multicolumn{2}{|c|}{ Coagulase } & \multirow{2}{*}{ Percentage $^{(*)}$} \\
\cline { 4 - 6 } & & & Positive & Negative & \\
\hline \multirow{2}{*}{ Staph.aureus } & Human & 14 & 14 & 0 & 31.11 \\
\cline { 3 - 6 } & Poultry & 5 & 5 & 0 & 14.29 \\
\hline \multirow{2}{*}{ Staph.chromogens } & Human & 0 & 0 & 0 & 0.00 \\
\cline { 2 - 6 } & Poultry & 2 & 0 & 2 & 5.71 \\
\hline \multirow{2}{*}{ Staph.hyicus } & Human & 2 & 2 & 0 & 4.44 \\
\cline { 2 - 6 } & Poultry & 2 & 2 & 0 & 5.71 \\
\hline
\end{tabular}


Ahmed et al., 2020

SVU-IJVS, 3 (1): 100-122

\begin{tabular}{|c|c|c|c|c|c|}
\hline \multirow{2}{*}{ Staph.hominis } & Human & 2 & 0 & 2 & 4.44 \\
\hline & Poultry & 2 & 0 & 2 & 5.71 \\
\hline \multirow{2}{*}{ Staph.haemolyticus } & Human & 3 & 0 & 3 & 6.67 \\
\hline & Poultry & 2 & 0 & 2 & 5.71 \\
\hline \multirow{2}{*}{ Staph.arlettae } & Human & 1 & 0 & 1 & 2.22 \\
\hline & Poultry & 2 & 0 & 2 & 5.71 \\
\hline \multirow{2}{*}{ Staph.warneri } & Human & 2 & 0 & 2 & 4.44 \\
\hline & Poultry & 2 & 0 & 2 & 5.71 \\
\hline \multirow{2}{*}{ Staph.pseudointermedius } & Human & 2 & 2 & 0 & 4.44 \\
\hline & Poultry & 0 & 0 & 0 & 0.00 \\
\hline \multirow[t]{2}{*}{ Staph.lentus } & Human & 2 & 0 & 2 & 4.44 \\
\hline & Poultry & 3 & 0 & 3 & 8.57 \\
\hline \multirow[t]{2}{*}{ Staph.epidermidis } & Human & 2 & 0 & 1 & 4.44 \\
\hline & Poultry & 0 & 0 & 0 & 0.00 \\
\hline \multirow[t]{2}{*}{ Staph.capitis } & Human & 1 & 0 & 1 & 2.22 \\
\hline & Poultry & 1 & 0 & 1 & 2.86 \\
\hline \multirow[t]{2}{*}{ Staph.vitulinus } & Human & 1 & 0 & 1 & 2.22 \\
\hline & Poultry & 2 & 0 & 2 & 5.71 \\
\hline \multirow[t]{2}{*}{ Staph.simulans } & Human & 0 & 0 & 0 & 0.00 \\
\hline & Poultry & 1 & 0 & 1 & 2.86 \\
\hline \multirow[t]{2}{*}{ Staph.auricularis } & Human & 1 & 0 & 1 & 2.22 \\
\hline & Poultry & 0 & 0 & 0 & 0.00 \\
\hline \multirow[t]{2}{*}{ Staph.cohnii } & Human & 3 & 0 & 3 & 6.67 \\
\hline & Poultry & 2 & 0 & 2 & 5.71 \\
\hline \multirow[t]{2}{*}{ Staph.saprophyticus } & Human & 2 & 0 & 2 & 4.44 \\
\hline & Poultry & 0 & 0 & 0 & 0.00 \\
\hline \multirow[t]{2}{*}{ Staph.schleiferi } & Human & 1 & 1 & 0 & 2.22 \\
\hline & Poultry & 2 & 2 & 0 & 5.71 \\
\hline \multirow[t]{2}{*}{ Staph.sciuri } & Human & 2 & 0 & 2 & 4.44 \\
\hline & Poultry & 2 & 0 & 2 & 5.71 \\
\hline
\end{tabular}


Ahmed et al., 2020

SVU-IJVS, 3 (1): 100-122

\begin{tabular}{|c|c|c|c|c|c|}
\hline Staph.xylosus & Human & 2 & 0 & 2 & 4.44 \\
\cline { 2 - 6 } & Poultry & 2 & 0 & 2 & 5.71 \\
\hline \multirow{3}{*}{ Staph.lugdunensis } & Human & 1 & 0 & 1 & 2.22 \\
\cline { 2 - 6 } & Poultry & 1 & 0 & 1 & 2.86 \\
\hline Staph.intermedius* & Human & 1 & 1 & 0 & 2.22 \\
\cline { 2 - 6 } & Poultry & 2 & 2 & 0 & 5.71 \\
\hline
\end{tabular}

(*) Percentage of each Staphylococcus species was calculated from the total number of isolates: poultry isolates $(n=35)$ and human isolates $(n=45)$

*the obtained isolates from poultry and human showed a significant correlation between each other's $\mathrm{R}=0.6$ and $\mathrm{P}<0.05$

Further that the antibiogram results showed that the least resistant species was Staph. simulans that was resistant to3 antibiotics (tetracycline, clindamycin and quinupristin/dalfopristin). While, Staph. aureus and Staph. lentus were the most resistant species to the 13tested antibiotics. While, methicillin-resistant Staphylococci were identified in 16isolates (8 as methicillin resistant coagulase positive Staphylococci (MRCoPS) and the other 8 were methicillin resistant coagulase negative Staphylococci (MRCoNS)). Vitek2 system also cleared that vancomycin resistant Staphylococcus species was detected in 14 isolates (Table 4)

The result of antibiogram showed in table (5) reveled that antimicrobial susceptibility profile of the 45 Staphylococcus isolates from human samples to 13 types of antibiotics. The high ratio of resistance to penicillin (84.4\%), clindamycin $(73 \%)$, tetracycline $(66.7 \%)$ and rifampicin (64.4\%). Also, penicillin and clindamycin recorded the high percentages of resistance (84.4\%) and (73\%) respectively. Whereas gentamicin and trimethoprim/sulfamethazole showed the low resistance $(26.7 \%)$ for both from tested antibiotics.

Further that the antibiogram results showed that the least resistant specie was Staph. auricularis that was resistant to 6 antibiotics (penicillin, clindamycin, vancomycin, ciprofloxacin, gentamicin and trimethoprim/sulfamethazole). On the other hand, Staph. aureus was the most resistant specie to the 13tested antibiotics. Methicillinresistant Staphylococci were detected in 24 isolates, (11were (MRCoPS) and the other 13 were (MRCoNS)). Vitek2 system also detected vancomycin resistant Staphylococcus species in 12 isolates.

Table (4): Distribution of Staphylococcus species isolated from poultry samples according to their species diversity and multidrug resistance pattern

\begin{tabular}{|c|c|c|c|c|c|c|c|c|c|c|c|c|c|}
\hline \multirow[b]{2}{*}{$\begin{array}{l}\text { Staphylococcus } \\
\text { sp. }(\mathrm{n}=\text { isolates })\end{array}$} & \multicolumn{13}{|c|}{ Antibiotics } \\
\hline & 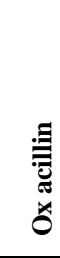 & 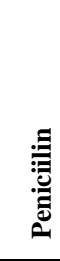 & 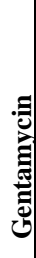 & 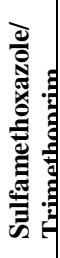 & 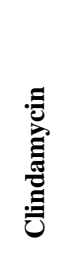 & 昰 & 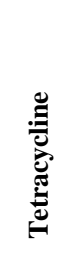 & 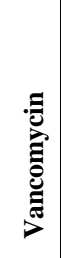 & 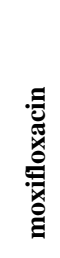 & 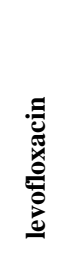 & 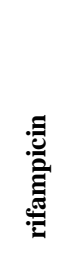 & 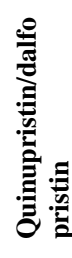 & 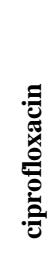 \\
\hline \multicolumn{14}{|c|}{ Resistance Pattern } \\
\hline Staph.aureus(5) & 4 & 4 & 1 & 2 & 5 & 4 & 4 & 2 & 2 & 2 & 5 & 2 & 3 \\
\hline
\end{tabular}


Ahmed et al., 2020

SVU-IJVS, 3 (1): 100-122

\begin{tabular}{|c|c|c|c|c|c|c|c|c|c|c|c|c|c|c|}
\hline \multirow{3}{*}{ Coagulase Positive } & Staph.hyicus(1) & 2 & 2 & 1 & 1 & 2 & 0 & 1 & 0 & 1 & 1 & 0 & 0 & $\overline{1}$ \\
\hline & Staph.schleiferi(2) & 1 & 0 & 0 & 0 & 0 & 2 & 1 & 1 & 2 & 2 & 1 & 1 & 2 \\
\hline & Staph.intermedius (2) & 1 & 2 & 1 & 1 & 1 & 0 & 2 & 1 & 0 & 0 & 0 & 2 & 0 \\
\hline \multirow[t]{13}{*}{ Coagulase Negative } & Staph.lentus (3) & 2 & 3 & 1 & 1 & 3 & 2 & 3 & 2 & 1 & 1 & 2 & 2 & 1 \\
\hline & Staph.hominis(2) & 0 & 0 & 0 & 0 & 0 & 0 & 1 & 0 & 1 & 1 & 0 & 0 & 1 \\
\hline & Staph.chromogens (2) & 0 & 2 & 0 & 0 & 0 & 2 & 2 & 1 & 0 & 0 & 2 & 0 & 0 \\
\hline & Staph.warneri(2) & 1 & 1 & 0 & 0 & 2 & 0 & 1 & 1 & 2 & 2 & 0 & 0 & 2 \\
\hline & Staph.haemolyticus(2) & 0 & 2 & 0 & 0 & 2 & 2 & 1 & 0 & 0 & 0 & 2 & 1 & 0 \\
\hline & Staph.arlettae(2) & 1 & 0 & 0 & 0 & 2 & 0 & 2 & 1 & 0 & 0 & 0 & 0 & 0 \\
\hline & Staph.sciuri(2) & 0 & 2 & 1 & 1 & 2 & 2 & 2 & 1 & 0 & 0 & 2 & 1 & 0 \\
\hline & Staph.xylosus(2) & 1 & 0 & 0 & 0 & 2 & 0 & 2 & 0 & 0 & 1 & 0 & 0 & 0 \\
\hline & Staph.vitulinus(2) & 1 & 2 & 1 & 1 & 0 & 0 & 2 & 2 & 1 & 1 & 0 & 0 & 1 \\
\hline & Staph.cohnii(2) & 0 & 0 & 0 & 0 & 2 & 2 & 1 & 0 & 0 & 0 & 2 & 1 & 1 \\
\hline & Staph.capitis(1) & 1 & 1 & 0 & 0 & 1 & 1 & 1 & 1 & 1 & 1 & 1 & 0 & 0 \\
\hline & Staph.simulans(1) & 0 & 0 & 0 & 0 & 1 & 0 & 1 & 0 & 0 & 0 & 0 & 0 & 0 \\
\hline & Staph.lugdunensis(1) & 1 & 1 & 0 & 0 & 1 & 1 & 1 & 1 & 1 & 1 & 0 & 1 & 1 \\
\hline Total & 35 & 16 & 22 & 6 & 7 & 26 & 18 & 28 & 14 & 12 & 13 & 17 & 11 & 13 \\
\hline
\end{tabular}

Table (5): Distribution of Staphylococcus species isolated from human samples according to their species diversity and multidrug resistance pattern

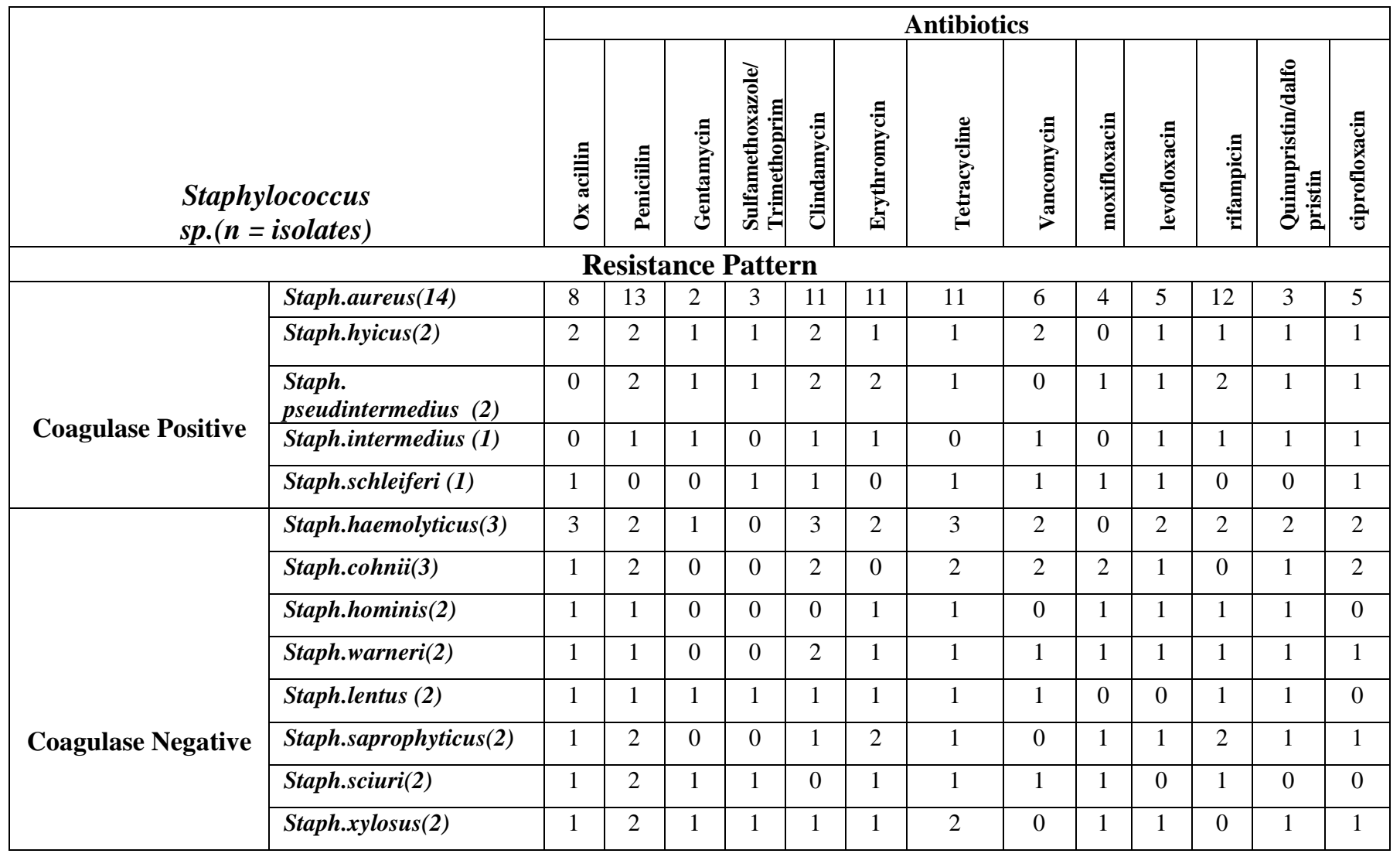


Ahmed et al., 2020

SVU-IJVS, 3 (1): 100-122

\begin{tabular}{|c|c|c|c|c|c|c|c|c|c|c|c|c|c|c|}
\hline & Staph.arlettae(1) & 1 & 1 & 1 & 1 & 1 & 1 & 1 & 1 & 0 & 1 & 1 & 1 & 1 \\
\hline & Staph.epidermidis(1) & 1 & 2 & 0 & 0 & 2 & 0 & 2 & 1 & 1 & 0 & 1 & 1 & 0 \\
\hline & Staph.capitis(1) & 0 & 1 & 0 & 0 & 1 & 1 & 0 & 1 & 1 & 0 & 1 & 0 & 1 \\
\hline & Staph.vitulinus(1) & 0 & 1 & 1 & 1 & 1 & 1 & 0 & 0 & 1 & 1 & 1 & 1 & 0 \\
\hline & Staph.auricularis(1) & 0 & 1 & 1 & 1 & 1 & 0 & 0 & 1 & 0 & 0 & 0 & 0 & 1 \\
\hline & Staph.lugdunensis(1) & 1 & 1 & 0 & 0 & 0 & 1 & 1 & 0 & 0 & 1 & 1 & 1 & 0 \\
\hline Total & 45 & 24 & 38 & 12 & 12 & 33 & 28 & 30 & 12 & 16 & 19 & 29 & 18 & 19 \\
\hline
\end{tabular}

\section{Results of PCR}

Ten Staphylococcus isolates were selected and classified into;5 isolates from poultry (Staph.lentus (1), Staph.aureus(2), Staph. lugdunensis(1) and Staph. warneri(1)) and 5 from human (Staph.cohnii(1), Staph.lentus, (1) Staph. aureus (2) and Staph. haemolyticus(1)) for testing by PCR. 16SrRNA gene primers confirmed the presence of Staphylococcal DNA in these isolates (Table 6\&Fig.1), also On the other hand,different antimicrobial resistance genes were detected in our isolates(ten isolates).mecA gene detected in 6 isolates devided into Staph. aureus(2), Staph. lugdunensis(1),Staph. warneri(1), Staph. cohnii (1)and Staph. lentus(1) and vanA gene were detected in 6 isolates (Staph aureus(4), Staph. lentus(1) and Staph. haemolyticus(1) while blaZ gene was detected in all isolates except Staph. lugdunensis and Staph haemolyticus, also cfr gene detected in 3 isolates (Staph aureus(2) and Staph lentus(1) (Table 6 and Fig.5,6,7and8) respectively. While sei and different virulence genes were detected in Staphylococcal isolates. Out of ten isolates coa gene was detected in four Staph. aureus isolates, hld gene detected in 6 isolates belonging to 3 Staph. aureus ,2 Staph.lentus and 1 Staph.haemolyticus and pvl gene detected in two Staph.aureus isolates (Table 6 and Fig.2,3 and4) respectively.

seh genes not detected in the tested samples. Staph. aureus,Staph.lentus and Staph. chonii harbored most of virulance and antimicrobial resistance genes respectively.

Despite of the harmony between aresults of Vitek system and PCR than conventional method as recorded in table(6) but PCR cleared a high accuracy in detection of mecA and vanA gene in human isolate no.(8), however Vitek system identified this isolate as vancomycin resistant strain only

\section{Table (6): The distribution of virulence genes and antimicrobial resistance genes in the}

\begin{tabular}{|c|c|c|c|c|c|c|c|c|c|c|c|c|}
\hline \multirow[b]{2}{*}{$\begin{array}{c}\text { Isolates } \\
\text { no. }\end{array}$} & \multirow[b]{2}{*}{ Source } & \multicolumn{2}{|c|}{ Vitek 2} & \multicolumn{2}{|c|}{ Conventional } & \multicolumn{7}{|c|}{ PCR } \\
\hline & & Species & $\begin{array}{c}\text { Resistance } \\
\text { genes }\end{array}$ & ORSAB & Coagulase & coa & hld & $p v l$ & mecA & blaZ & vanA & $c f r$ \\
\hline 1 & Poultry & Staph.lentus & $\begin{array}{l}\text { vancomycin } \\
\text { resistant }\end{array}$ & +ve & -ve & - & + & - & - & + & + & + \\
\hline 2 & Poultry & Staph.aureus & $\begin{array}{l}\text { vancomycin } \\
\text { resistant }\end{array}$ & -ve & +ve & + & + & + & + & + & + & - \\
\hline 3 & Poultry & Staph.aureus & $\begin{array}{l}\text { vancomycin } \\
\text { resistant }\end{array}$ & -ve & $+\mathrm{ve}$ & + & - & - & - & + & + & - \\
\hline
\end{tabular}


Ahmed et al., 2020

SVU-IJVS, 3 (1): 100-122

\begin{tabular}{|c|c|c|c|c|c|c|c|c|c|c|c|c|}
\hline 4 & Poultry & Staph.lugdunensis & $\begin{array}{c}\text { methicillin } \\
\text { resistant }\end{array}$ & $+\mathrm{ve}$ & $-\mathrm{ve}$ & - & - & - & + & - & - & - \\
\hline 5 & poultry & Staph.warneri & $\begin{array}{c}\text { methicillin } \\
\text { resistant }\end{array}$ & $-\mathrm{ve}$ & $-\mathrm{ve}$ & - & - & - & + & + & - & - \\
\hline 6 & human & Staph.cohnii & $\begin{array}{c}\text { methicillin } \\
\text { resistant }\end{array}$ & $+\mathrm{ve}$ & $-\mathrm{ve}$ & - & - & - & + & + & - & - \\
\hline 7 & human & Staph.lentus & $\begin{array}{c}\text { methicillin } \\
\text { resistant }\end{array}$ & $-\mathrm{ve}$ & $-\mathrm{ve}$ & - & + & - & + & + & - & - \\
\hline 8 & human & Staph.aureus & $\begin{array}{c}\text { vancomycin } \\
\text { resistant }\end{array}$ & $-\mathrm{ve}$ & $+\mathrm{ve}$ & + & + & + & + & + & + & + \\
\hline 9 & human & Staph.aureus & $\begin{array}{c}\text { vancomycin } \\
\text { resistant }\end{array}$ & $+\mathrm{ve}$ & $+\mathrm{ve}$ & + & + & - & - & + & + & + \\
\hline 10 & human & Staph.haemolyticus & $\begin{array}{c}\text { vancomycin } \\
\text { resistant }\end{array}$ & $-\mathrm{ve}$ & $-\mathrm{ve}$ & - & + & - & - & - & + & - \\
\hline
\end{tabular}

\section{tested isolates}
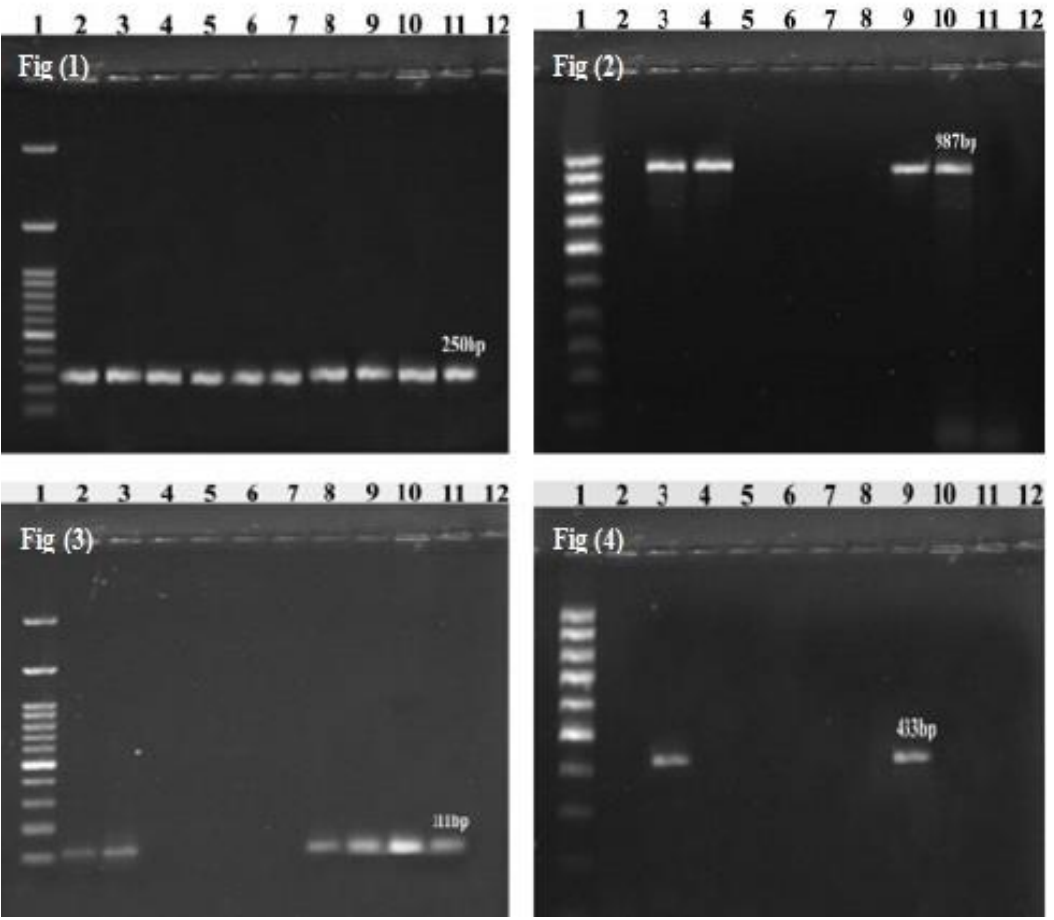

Fig (1): Agarose gel electrophoresis of $16 s r R N A$ gene amplification at 250b, Lane 1:

Ladder, (100bp), Lanes 2 to 6: positive poultry isolates, Lanes 7 to 11: positive human isolates.

Fig (2): Agarose gel electrophoresis of coa gene amplification at 987bp, Lane 1: Ladder (100bp), Lanes 2 to 6: poultry isolates, included lane 3 and 4 were positive isolates, Lanes 7 to 11: human isolates, included lanes 9 and 10 were positive isolates.

Fig (3): Agarose gel electrophoresis of hld gene amplification at 111bp, Lane 1: Ladder (100bp), Lanes 2 to 6: poultry isolates, included lane 2 and 3 were positive isolates, Lanes 7 to 11: human isolates, included lane 8,9,10,11 were positive isolates.

Fig (4): Agarose gel electrophoresis of $P v l$ gene amplification at $433 \mathrm{bp}$, Lane 1: Ladder (100bp), Lanes 2 to 6: poultry isolates, included lane 3 was positive isolate, Lanes 7 
to 11: human isolates, included lane 9 was positive isolate
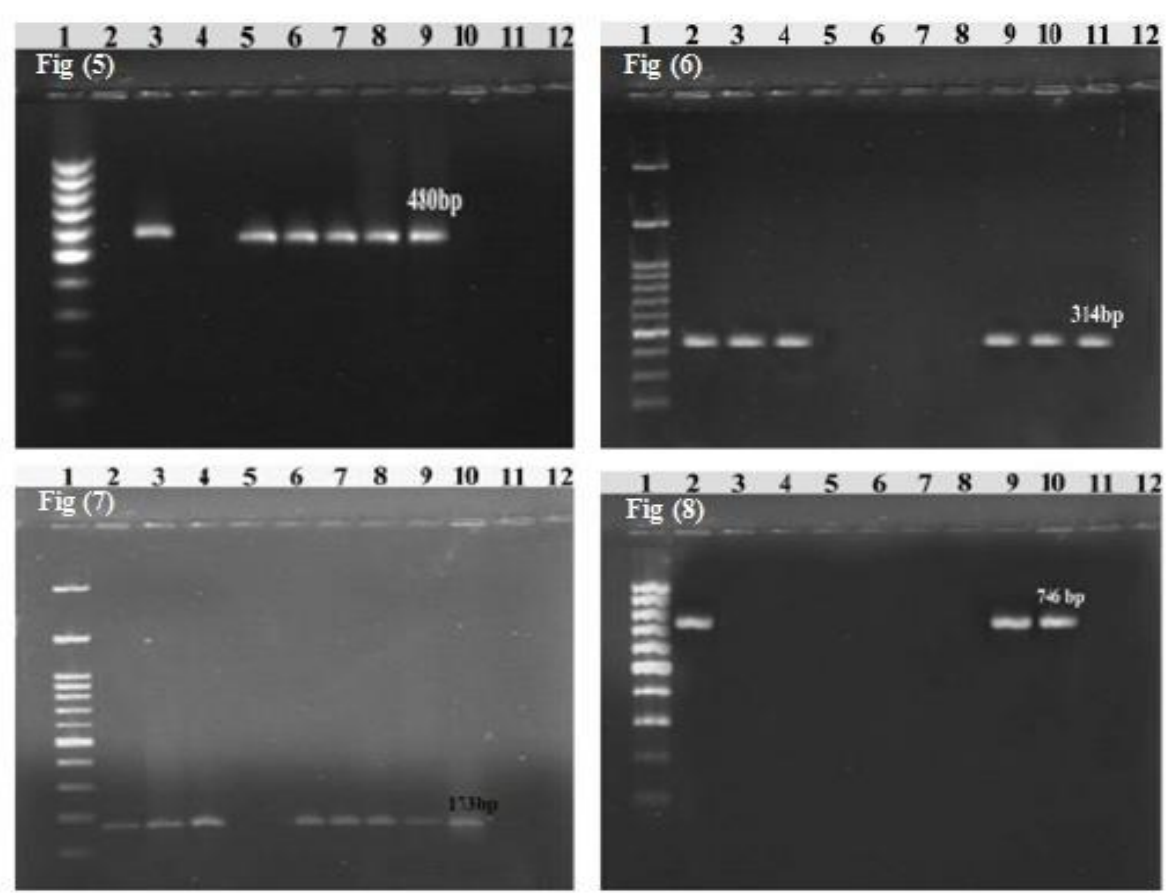

Fig (5): Agarose gel electrophoresis of mecA gene amplification at 480, Lane 1: Ladder (100bp), Lanes 2 to 6: poultry isolates, included lane 3,5 and 6 were positive isolates, Lanes 7 to 11: human isolates, included lane 7, 8 and 9 were positive isolates

Fig (6): Agarose Gel electrophoresis of van $A$ gene amplification at $314 \mathrm{bp}$, Lane 1: Ladder (100bp), Lanes 2 to 6: poultry isolates, lane 2,3and 4 positive, Lanes 7 to 11: human isolates, lane 9,10 and 11postive.

Fig (7): Agarose gel electrophoresis of BlaZ gene amplification at $173 \mathrm{bp}$, Lane 1: Ladder (100bp), Lanes 2 to 6: poultry isolates, included lane 2,3,4,6 were positive isolates, Lanes 7 to 11: human isolates, included lane 7,8,9,10were positive isolates.

Fig (8): Agarose gel electrophoresis of cfr gene amplificationat746bp, Lane 1: Ladder (100bp), Lanes 2 to 6: poultry isolates, included lane 2 positive, Lanes 7 to 11: human isolates, included lane 9 and lane 10 positive.

Table (7): Accession number of isolated Staphylococcus spp

\begin{tabular}{|c|c|c|c|}
\hline $\begin{array}{c}\text { Isolate } \\
\text { No. }\end{array}$ & Isolate species & Source & Accession number \\
\hline 1 & Staph lentus & Poultry & OM976507 \\
\hline 2 & Staph.aureus & poultry & OM920073 \\
\hline 3 & Staph.aureus & poultry & OM920074 \\
\hline 7 & Staph lentus & human & OM976506 \\
\hline 8 & Staph aureus & human & OM920070 \\
\hline
\end{tabular}




\begin{tabular}{|c|c|c|c|}
\hline 9 & Staph.aureus & human & OM918375 \\
\hline 12 & Staph.aureus & human & OM918376 \\
\hline
\end{tabular}

\section{Results of phylogeny}

The blasting of our sequncing results on gene bank confirmed the identification results of Vitek2 system.As well,the results of phylogenetic analysis showed that our Staph.aureus strains isolated from both poultry and human cleared high similarities between each other ranged from (99.7-99.9\%)(Tabe.7\& Fig.9) and grouped with refernce isolates obtained from gene bank; (LN871053) from wound in India , (MN078268) water in India, (KR085871) water with range (94.599.9\%)(Fig.9\&Supp.tabel.1) . In addition to, our Staph.lentus isolates showd identity with percentage $(96.6 \%)$ (Tabe.7 Fig.9),also group with refernce isolates from poultry houses in Bangaladesh (MN701067) and soil in Korea (MF948914) with percentage(95.699.2\%)(Fig.9\&Supp.tabel.1)

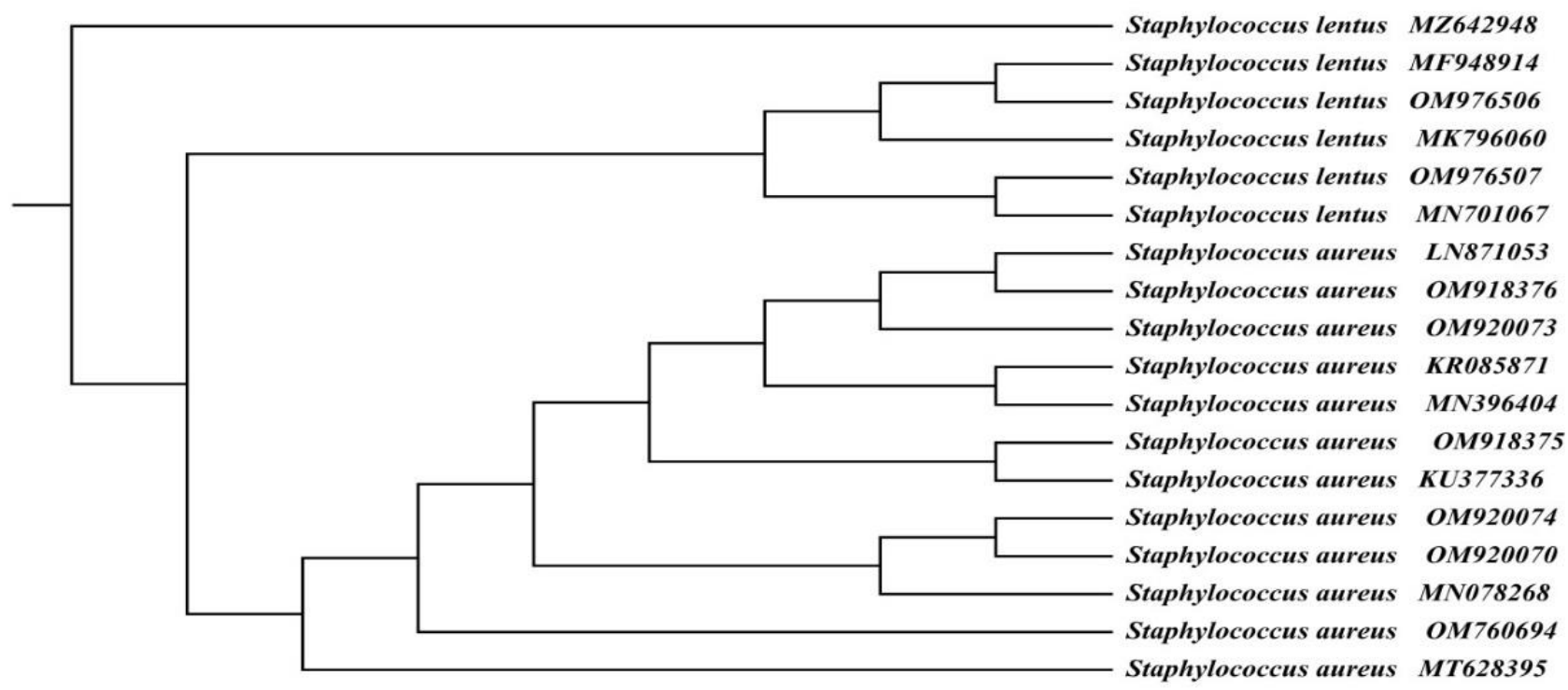

Figure9: Phylogenetic Tree of the entire nucleotide sequence of 16SrRNAgene of our Staphylococcus spp. isolates compared with reference strains regained from Gene Bank

\section{Discussion}

Earlier studies have revealed that Staphylococci are a common inhabitant of the chicken intestinal tract (Lee et al.,2018). Though, their presence in the intestines can have dual roles as commensals and pathogens (Rosenstein and Gotz, 2013). Similarly Staphylococci can be found as natural inhabitants of the skin of humans and other animals, they may also cause infections in those same tissues as well as other diseases. In poultry, researches have also proved an association of both coagulase positive Staphylococci as well as coagulase negative Staphylococci with poultry diseases (AlRubaye et al., 2015; Nazia et al., 2015). The problem of infections caused by these micro-organisms cannot be treated with common antibiotics (Phillips et al., 2004)

There are a different traditional diagonistic methods for identification of 
Staphylococcus spp. such as growing on mannitol salt agar, coagulase and acetoin production (Kateete et al., 2010), in this study Staphylococcus species recovered from $35 \%$ and $45 \%$ in poultry and human samples respectively on mannitol salt agar (Table 2). Higher percentage of Staphylococcus spp. in poultry farms $(52.5 \%)$ were observed by Onaolapo et al. (2017)lower incidence (10.8\%)were recorded by Marek et al.(2016). Ghias et al.(2016)isolated Staphylococcus with high rate $(55 \%)$ from pus samples of patient's skin.

Coagulase test divided the poultry and human isolates into $31.4 \%$ and $44.4 \%$ coagulase positive and $68.6 \%$ and $55.6 \%$ were coagulase negative respectively. Higher results were observed by Islam et al. (2014)and Ghias et al. (2016).

Oxacillin Resistance Screening Agar Bas (ORSAB) improved the recovery of methicillin-resistant Staphylococci in $37.14 \%$ and $42.22 \%$ from Staphylococcal isolates from poultry and human respectively (Table 2). In comparison to our result, Simor et al.(2001) recorded a higher predictive value for isolation of MRSA from different clinical samples $(76 \%)$.

The elevation in ratio of contamination by pathogenic species of Staphylococcus might have resulted to the contamination of hatcheries, farm surroundings and by tools used in the farms, also it has been recorded that the isolation of Staphylococci in poultry and its products are often connected to low hygienic methods during slaughtering, cutting, storage and shopping(Karmi, 2013).

The phenotypic differentiation of Staphylococcus spp. is considered a complicated case due to the absence of precise biochemical markers. Nevertheless, phenotypic analyses can't reach to complete identification for Staphylococcus species, also, this method are relatively time consuming and most importantly, difficult to analyze results. So, the use of automated devices such as Vitek 2 system has become routine in human and veterinary microbiology fields to overcome the traditional methods problems(Sasaki et al., 2010).

In the current study, by using VITEK 2 system, poultry isolates could be differentiated into 17 Staphylococcal species, the highly identified coagulase positive specie was Staph.aureus (14.2\%) and coagulase negative specie was Staph.lentus $(8.5 \%)$ (Table 3), this result in accordance with Wieliczko et al.(2002)who found that the most frequently coagulase positive specie was Staph.aureus and among coagulasenegative species were Staph.lentus, Staph.xylosus and Staph.cohnii. On the other hand,Marek et al.(2016)detected the most isolated species were Staph.cohnii $(23.50 \%)$, Staph.aureus $(15.89 \%)$ and Staph.lentus (13.90\%).However Coagulase negative Staphylococci are less pathogenic than S. aureus (Livermore,2000) but they was were isolated from infected chickens with cellulitis, granulomas in the liver and lungs , gangrenous dermatitis and abscesses in chickens(Linares and Wigle 2001 and Stępień-Pyśniaket al.,2017)

Human isolates could be differentiated into 19 Staphylococcal species, the highly identified species were Staph.aureus 14isolates $(31.1 \%)$ followed by Staph.haemolyticus and Staph.cohnii 3 isolates for each species ( $6.7 \%$ for each) by VITEK 2 system (Table 3).Higher results were obtained by Abd El-Tawab et al.(2017)who isolated Staph.aureus from human samples with percentage $67.5 \%$. On the other hand, Delmas et al.(2008)detected the most frequently occurring species were Staph.epidermidis (20\%) followed by Staph.saprophyticus and Staph.warneri (10\% for each).The difference in percentage of Staphylococcus 
isolation may be due to different reasons consist of (human and animal sources), geographical situation, numbers of samples and a variation routine of isolation (Fagundes and Oliveira, 2004).

The data recorded in table (4 and 5) demonstrated that the isolates of poultry and human showed multidrug resistance (resistant to $\geq 3$ class of antibiotics) high percentages of resistance were observed to tetracycline, clindamycin, penicellin and gentamycin. The high most resistant specie was Staph.aureus, it showed a resistance for 13 types of antimicrobials, this result reinforced by Onaolapo et al. (2017)who used different biochemical parameters such as disk diffusion, microgen Staph. kit and other tests and found their isolates resisted more than 3 family of antibiotics. Nearly related results of antimicrobial susceptibility have been recorded by Leonard and Markey (2008); Otalu et al.(2011); Pesavento et al.(2007) andWaters et al.(2011).

Vitek2 system detected methicillinresistant Staphylococci in $45.7 \%$ of poultry isolates and $53.3 \%$ of human isolates (Table 4\&5) while ORSAB detected methicillin-resistant Staphylococci in $37.1 \%$ of poultry isolates and $42.2 \%$ of human isolates (Table 2). This result confirmed that Vitek2 system is more accurate in the detection of resistant strains of Staphylococcus than ORSAB(Sukru et al., 2018).Moreover, it was more rapid in getting of the results (12hrs) and overcome the false negative results than ORSAB(24hrs)(Malaviolle et al., 2008).

PCR considered a gold stander in identification Staphylococcus spp. and became more essential to overcome the difficulties of conventional methods. In our results 16SrRNA gene confirmed the presence of Staphylococcal DNA in our isolates (Table 6 and Fig.1). The role of this gene was reinforced by many authors (Ghebremedhin et al.,2008 and Johnson et al.,2016), also, coagulase gene (coa gene) has a title role in identification of these species in this study,it detected 4 Staph.aureus isolates (Table 6 and Fig.2), this result supported by Vintov et al. (2003) who found that coa gene can be used for research purposes to explored diversity and polymorphism of Staphylococcus, also, Bharadwaz et al. (2015)decided that coa gene was considered as a marker for identification of Staph.aureus strains and other novel species for instance Staph.intermedius, Staph.delphini, Staph.shleiferi as coagulase positive species.

Different virulence genes were harbored by Staphylococcus spp., one of the most important virulence gene was haemolysin gene $(h l d)$ which is exoproteins that are produced by Staphylococci, haemolysin is responsible for the increased dissemination and virulence of these species. In present study, hld gene detected in 6 isolates belonging to different Staphylococcus spp. Staph. aureus and Staph. lentus the most species harbored this gene (Table 6 and Fig.3). Abdalrahman et al. (2015) found the incidence of hld gene was $(75.6 \%)$ in the 168 Staph. aureus isolates from poultry. Also, Rossato et al. (2018) detected hla gene in $87.6 \%$ from 177 nosocomial MRSA strains isolated from patients.

Panton-Valentine leukocidin $(p v l)$ was a cytotoxin gene and has a major role in the pathogenicity of this bacteria, this toxin form pores in the membrane of host defense cells, and be able to cause severe necrotic pneumonia, tissue infections furthermore to its ability to cause life threatening and associated with community-acquired MRSA infections (Motamedi et al., 2015).Kraushaar and Fetsch(2014)elucidated $p v l$ gene incidence among (MRSA) in retail poultry meat and slaughter employee and emphasized the 
impact of this animal reservoir on human health care. In this study, $p v l$ gene was detected in Staph.aureus (one isolate from each poultry and human isolates)(Table 6 and Fig.4), Tawfiq (2018)detected $p v l$ gene in three isolates from fresh chicken and Jackson et al. (2013)detected $p v l$ gene in one Staph.aureus isolated from retail beef, in addition to Durand et al.(2006) and Thabit et al. (2017) detected $p v l$ gene in community-acquired infection isolates.

In the existing study multidrug resistance was perceived, a number of genes have been clarified for detection of antimicrobial resistance in different species of Staphylococcus (Table 6). Resistance to methicillin is intended by the existence of the mecA gene encoding PBP2a which has a very low affinity to $\beta$-lactam antibiotics (Rice, 2012).Wendlandt et al.(2015) reported the implication of methicillin resistant Staph.aureus (MRSA) in poultry, as it was the utmost consumed protein responsible for wide spreading of MRSA among humans, that could be fatal and associated with multi-drug resistance.

Our results cleared that mecAgene was detected in 3 isolates out of 5 from each of poultry and human samples belonging to different species as Staph.aureus, Staph. lugdunensis, Staph. warneri, Staph. cohnii and Staph. lentus by PCR. Staph.aureus was the most specie harbored this gene (Table 6and fig.5). This results supported by Ali et al. (2017) and Abdalrahman et al. (2015) who recorded that incidence of mecA gene in Staph.aureus isolates was $1.2 \%$. Osman et al. (2016) conveyed the mecA gene in different species (Staph. lugdunensis; Staph. haemolyticus; Staph. hominus and Staph. lentus) isolated from poultry. Al-Muhanna (2014) confirmed by PCR that all CoNS isolated from poultry carried mec $A$ gene, also, Mulders et al. (2010) detect mecAgene in 26 out of 466
$(5.6 \%)$ Staph.aureus isolates of individuals. Coelho et al. (2007)found that 12 out of 80 Staph.aureus isolates (15\%) of human have mecA gene.

The presence of MRSA in both poultry and human isolates is a zoonotic issue among animals and humans through direct contact, environmental contamination, and contaminated animal products (Aqib et al.,2017 and Erwin et al.,2014).

Molecular detection became a necessary tool because methicillin resistance is often heterogeneously expressed in vitro and provides consistent results because the protocol is basically standardized and has progressed as an proficient tool for epidemiological

investigations(Strommenger et al., 2006), these result supported our results which cleared that PCR detected the mecA gene in isolate that not detected as methicillin-resistant Staphylococciby in the vitek2. Also, these results mean that macA gene was present but hasn't any expression (Hoopes, 2008). However, Shan et al. (2016) asserted the role of Vitek in predicting by MRSA even if the accuracy rate is not perfectly reached $100 \%$.

Higher mortality, greater morbidity were recorded in patients infected with methicillin-resistant Staphylococci, they utilize more healthcare resources compared with those who have infections instigated by methicillin-susceptible Staphylococci(Itani, 2016).The previous data emphasized the increasing role of Staphylococci in poultry infections, which recommended that the safety risks associated with their occurrence in the food consumed by humans, induced hospital infections with a high mortality rate (De Silva et al., 2002; Piette and Verschraegen, 2009). 
Vancomycin resistant Staph.aureus (VRSA) were a protuberant pathogens that cause a wide range of infections in different hosts(Grundmann et al., 2010). These strains convey the vanA gene that responsible for depressing the cell wall affinity for Vancomycin(Sibbald et al., 2006).By using PCR assay, we detected vanA genein3out of 5 isolates from each of poultry and human samples(Table 6and fig.6).Martins et al. (2013)detected vanA gene in 3 samples out of 15 of chilled industrialized uncooked chicken parts andOkolie et al.(2015)found vanA in 22isolates out of 155 from chicken carcasses. On other hand,Saadat et al. (2014)detected vanA in $34 \%$ of clinical isolates in hospital, while Khudaier(2018)detected vanA in 4 human isolates out of 163 .

Taponen and Pyörälä(2009)reported that the utmost communal mechanism of Staphylococcus resistance to antibiotics is the production of $\beta$-lactamases due to the presence of blaZgenethat coded for an alteration of penicillin-binding protein $2 \mathrm{a}$ which reduced the affinity for $\beta$-lactam antibiotics. The Clinical and Laboratory Standards Institute (CLSI) recommending the detection of blaZ gene specially in infected cases treated previously with penicillin (Testing and Testing, 2016).Results obtained in this study indicate the presence of blaZ gene indifferent Staphylococcal spp. obtained from poultry and human samples(Table 6 and Fig.7), this results related to Ferreira $e$ tal.(2017);Mkize(2016); Pyzik et al.(2019) andWhichard et al. (2007).

Other antimicrobial resistant gene was $c f r$ gene was detected in different Staphyloccous spp. in our isolates as Staph.aureus and Staph.haemolyticus(Tabel 6 and Fig 8). A different mechanism of linezolid resistance has recently been described in veterinary staphylococcal isolates. The mechanism is non mutational and includes attainment of a natural resistance gene, $c f r$ (Kehrenberg et al.,2007).This gene encodes an rRNAmethyl-transferase which modifies the adenine at position 2503 in 23SrRNA(Kehrenberg et al., 2005).It confers a resistance not only to linezolid, but also to phenicols, lincosamides, pleuromutilinsand streptograminA antibiotics (Long et al., 2006). Toh et al. (2007) stated the first cfr-mediated, linezolid-resistant clinical isolate of MRSA. Wang et al. (2013) detected the $c f r$ gene in Staph.haemolyticus

It is worth to record that surprisingly a high percentage of strains were resisted to several types of antibiotics used in this study. From a clinical view this is a vital observation, as resistant bacteria can transmit genes coding for antibiotic resistance to other bacteria by transduction, conjugation or transformation. This may lead to a spreading antibiotic resistance rapidly in the Staphylococcus population(Marek et al., 2016).These information can be used to inform public health official to enforce judicious use of antimicrobial agents in human and veterinary medicine (Cummings et al., 2013).Otto (2013) recorded that coagulase negative Staphylococcus supposed to act as reservoirs for antibiotic resistance genes.

Several studies confirmed pathogenicity of coagulase positive Staphylococcus( Livermore et al.,2000, Youssef and Hamed, 2012) but the most we can talk about in this study multidrug drug resistance of coagulase negative and its possession to different virulence and antimicrobial resistant genes, these results supported by Becker et al.(2014) who cleared that Strains of coagulase negative of both animal and human origins are supposed to attend as main reservoirs of antimicrobial resistance genes. These genes are frequently cited on mobile genetic elements, permitting their 
horizontal transfer to pathogenic

Staphylococci (Resch et al. 2008).

The results of 16SrRNA gene sequencing in Stah.aureus and Staph.lentus isolated from both poultry and human showed that a high similarity in sequence of the same species obtained from poultry and human isolates with identity range (99.9$100 \%$ )and the surprise is that most of our isolates were grouped with reference strains obtained from environment like soil and water with identity(95.6-100\%) and this may be elucidated these resemblances between our isolates of the same spp because they have the same source. Pan and $\mathrm{Yu}$ (2014) supported these results and clarified that several types of microorganisms existent in poultry intestines largely depend on their diet and the environment in which they live besides Staph.ylococcal spp. were have a double roles as commensals and pathogen for Conflict of interest

The authors declare that they have no conflict of interest.

\section{References}

Aarestrup, F.M., Agers, L.Y., Ahrens, P., Lrgensen, J.C., Madsen, M. and Jensen, L.B. Antimicrobial susceptibility and presence of resistance genes in staphylococci from poultry. Vet. Microbiol., 74(4), 353-64 (2000)

Abd El-Tawab AA, Hofy FI, Mohamed SR, Amin $\mathrm{SH}(2017)$. Characterization of Methicillin Resistance Staphylococcus aureus isolated from chicken and human. Benha Veterinary Medical Journal, 32: 132-137.

AbdalrahmanL, Stanley A, Wells H, Fakhr $\mathrm{M}(2015)$. Isolation, virulence, and antimicrobial resistance of methicillin-resistant Staphylococcus aureus (MRSA) and methicillin sensitive Staphylococcus aureus (MSSA) strains from Oklahoma retail poultry meats. International journal of environmental research and public health, 12: 6148-6161.

Adesiji YO, Deekshit VK, Karunasagar I(2014). Antimicrobial-resistant genes associated with Salmonella spp. isolated from human, poultry, and seafood sources. Food science \& nutrition, 2: 436442.

Alfatih YM, Idris AB, Hassan HG, Nour EO, Elhaj NM, El-Zaki SE, Eldirdery MM, Ali RH, Ibrahim both poultry and human (Rosenstein and Gotz, 2013) so the transmission between two hosts can be occurred.

\section{Conclusion}

Finally, we concluded that ViteK system played important role in identification of Staphylococcus spp and their susptability to different antimicrobials. PCR gave a good screening for virulance and antimicrobials genes in, also sequencing of $16 S r R N A$ cleared the releation between our isolates. So, we recommended decreasing the unwarranted use of antimicrobials in poultry production and educate people about the use of antibiotics

\section{Acknowledgments}

The authors are thankful to all staff members in Microbiology Department, Faculty of Veterinary Medicine, South Valley University, Egypt, and Molecular unit in Assiut University Hospital, Egypt

NY, Elegail AM, Salih MA(2018). Detection of a novel mutation G511T in the 530 loop in $16 \mathrm{~S}$ rRNA in multi drugs resistant Mycobacterium tuberculosis isolated from Sudanese patients. BioRxiv.;1:497628.

Al-Muhanna, A. S., Al-Hilu, S. A., and Alzuhairi, M. A. (2014). Characterization of coagulasenegative, oxacillin resistant Staphylococcifrom patients undergoing catheter related infections. Eur. J. Exp. Biol., 4(3):774-778

Al-Rubaye A.A., Couger M.B., Ojha S., Pummill J.F., Koon J.A., 2nd, Wideman R.F., Jr., Rhoads D.D. Genome analysis of Staphylococcus agnetis, an agent of lameness in broiler chickens. PLoS One. 2015;10:e0143336

Aqib AI, Ijaz M, Anjum AA, Malik MAR, Mehmood K, Farooqi SH, et al. Antibiotic susceptibilities and prevalence of Methicillin resistant Staphylococcus aureus (MRSA) isolated from bovine milk in Pakistan. Acta Trop. 2017; 176:168-172.

Ali Y, Islam MA, Muzahid NH, Sikder MOF, Hossain MA, Marzan LW(2017). Characterization, prevalence and antibiogram study of Staphylococcus aureus in poultry. Asian Pacific Journal of Tropical Biomedicine, 7: 253-256. 
Becker K, Heilmann C, Peters G(2014). Coagulasenegative Staphylococci. Clinical microbiology reviews, 27: 870-926.

BharadwazM, Kalita M, Gogoi K, Dey T, Ozah D, Das R, Saikia I, Unni BG(2015) . Preliminary characterization of blood coagulase protein from a Staphylococcus strain. Int. J. Pharm. Pharm. Sci., 6: 151-155.

Chajęcka-Wierzchowska W, Zadernowska A, Nalepa B, Sierpińska M, Łaniewska-Trokenheim Ł (2015) . Coagulase-negative Staphylococci (CoNS) isolated from ready-to-eat food of animal originphenotypic and genotypic antibiotic resistance. Food microbiology, 46: 222-226.

Coelho SdM, Moraes R, Soares LdC, Pereira I, Gomes L, de Souza M(2007) . Resistance pattern and detection of mecA gene in oxacillin-resistant isolates of Staphylococcus aureus and Staphylococcus intermedius from animal and human samples. Ciencia Rural (Brazil).

CummingsKJ, Perkins GA, Khatibzadeh SM, Warnick LD, Altier C(2013) . Antimicrobial resistance trends among Salmonella isolates obtained from dairy cattle in the northeastern United States, 2004-2011. Foodborne pathogens and disease, 10: 353-361.

Cunha M(2009) . Staphylococcus aureus and bovine mastitis: a public health problem. Cross Infections: Types, Causes and Prevention, eds J. Dong and X. Liang (New York, NY: Nova Science Publiseh rs), 117-128.

De Silva G, Kantzanou M, Justice A, Massey R, Wilkinson A, Day N, PeacockS(2002). The ica operon and biofilm production in coagulasenegative Staphylococci associated with carriage and disease in a neonatal intensive care unit. Journal of clinical microbiology, 40:382-388.

Delmas J, Chacornac JP, Robin F, Giammarinaro P, Talon R, Bonnet R(2008) . Evaluation of the Vitek 2 system with a variety of Staphylococcus species. Journal of clinical microbiology, 46: 311-313.

ht MC, Forey F, Liassine N, Wenger A, Kikuchi K, Lina G, Vandenesch F(2006). Detection of new methicillin-resistant Staphylococcus aureus clones containing the toxic shock syndrome toxin 1 gene responsible for hospital-and community-acquired infections in France. Journal of clinical microbiology, 44: 847-853.

Erwin V, Jan K. Livestockassociated Staphylococcus aureus CC398: Animal reservoirs and human infections. Infect Genet Evol. 2014;21(1):523-30

Fagundes H, Oliveira CAF(2004) . Infecções intramamárias causadas por Staphylococcusaureus e suas implicações em paúde pública. Ciência Rural, 34: 1315-1320.
Ferreira AM, Martins KB, Silva VRd, Mondelli AL, Cunha MdLRd(2017) . Correlation of phenotypic tests with the presence of the blaZ gene for detection of beta-lactamase. brazilian journal of microbiology, 48: 159-166.

Fijałkowski K, Peitler D, Karakulska J(2016) . Staphylococci isolated from ready-to-eat meatidentification, antibiotic resistance and toxin gene profile. International journal of food microbiology, 238: 113-120.

Gharajalar SN, Shahbazi P(2018) . Antimicrobial susceptibility patterns of biofilm forming Staphylococcus aureus isolated from pigeon external ocular infections. Journal of Exotic Pet Medicine, 27:81-84.

GhebremedhinB, LayerF,Ko"nig W, Ko"nigB(2008) . Genetic Classification and Distinguishing of Staphylococcus Species Based on Different Partial gap.J.Clinc.Microbiology, 46(3): 1019-1025

Ghias W, Sharif M, Yazdani FA, Rabbani M(2016) . Isolation and identification of Methicillin and Vancomycin resistance Staphylococcus aureus from pus samples of injured skin patients in Lahore, Pakistan. Biomed Lett, 2:103-112.

Götz F, Bannerman T, SchleiferK(2006) . The genera Staphylococcus and Macrococcus. The prokaryotes. Dworkin M, Falkow S, Rosenberg E, Schleifer KH, Stackebrandt (eds) .

Grundmann H, AanensenDM, Van Den Wijngaard CC, Spratt BG, Harmsen D, Friedrich AW, Group ESRLW(2010) . Geographic distribution of Staphylococcus aureus causing invasive infections in Europe: a molecular-epidemiological analysis. PLoS medicine, 7:e1000215.

Hoopes L(2008) . Introduction to the gene expression and regulation topic room. Nature Education, 1(1): 160

Jackson CR, Davis JA, Barrett JB(2013) . Prevalence and Characterization of MethicillinResistantStaphylococcusaureusIsolates from Retail Meat and Humans inGeorgia. Journal of Clinical Microbiology, 4(51) : 1199-1207

JohnsonJE, ZemanickTE, AccursoJF, WagnerDB, RobertsonEC, Kirk HarrisJ(2016) .Molecular Identification of Staphylococcusaureus in Airway Samples from Children with Cystic Fibrosis.PLoS One, 11(1): e0147643

Islam NN, Akter M, Farzana Z, Kader AJB, Uddin I, Siddiki A, Kamaruddin K(2014) . Detection of Staphylococcus aureus in frozen chicken rinse through bacteriological and nuc gene specific PCR methods and their drug resistance patterns in Southern Chittagong, Bangladesh. Research Journal of Microbiology, 9: 251-264. 
Itani KM(2016) . MRSA and complicated skin and soft tissue infections, MRSA. CRC Press, pp. 7289 ..

Jarraud S, Mougel C, Thioulouse J, Lina G, Meugnier H, Forey $\mathrm{F}$, Nesme $\mathrm{X}$, Etienne J, Vandenesch F(2002) . Relationships between Staphylococcus aureus genetic background, virulence factors, agr groups (alleles), and human disease. Infection and immunity, 70: 631-641.

Kadariya J, Smith TC, Thapaliya D(2014) . Staphylococcus aureus and Staphylococcal foodborne disease: an ongoing challenge in public health. BioMed research international 2014.

Karmi M(2013) . Prevalence of methicillin-resistant Staphylococcus aureus in poultry meat in Qena, Egypt. Veterinary World 6.

Kateete DP, Kimani CN, Katabazi FA, Okeng A, Okee MS, Nanteza A, Joloba ML, Najjuka FC(2010) . Identification of Staphylococcus aureus: DNase and Mannitol salt agar improve the efficiency of the tube coagulase test. Annals of clinical microbiology and antimicrobials, 9:23.

Kehrenberg C, Aarestrup FM, Schwarz S. IS21558 insertion sequences are involved in the mobility of the multiresistance gene cfr. Antimicrob Agents Chemother 2007; 51:483-487.

Kehrenberg C, Schwarz S(2006) . Distribution of florfenicol resistance genes fexA and cfr among chloramphenicol-resistant Staphylococcus isolates. Antimicrobial agents and chemotherapy, 50: 11561163.

Kehrenberg C, Schwarz S, Jacobsen L, Hansen LH, Vester B(2005) . A new mechanismfor chloramphenicol, florfenicol and clindamycin resistance: methylation of $23 \mathrm{~S}$ ribosomal RNA at A2503. Mol. Microbiol., 57: 1064-1073.

Khudaier BY(2018). Molecular Detection of vanA Gene in Staphylococcus aureus Isolated from Stool Samples in North India. basrah journal of science, 36: 26-38.

Kim J, Kim M(2017) . Study on the Infection of Staphylococcus aureus and Diagnostic Methods in Food.

Kraushaar B, Fetsch A(2014). First description of PVL-positive methicillin-resistant Staphylococcus aureus (MRSA) in wild boar meat. International journal of food microbiology, 186: 68-73.

Lee, A., de Lencastre, H., Garau, J. et al. (2018)Methicillin-resistant Staphylococcus

aureus. Nat Rev Dis Primers 4, 18033 https://doi.org/10.1038/nrdp.2018.33

Leonard F, Markey B(2008) . Meticillin-resistant Staphylococcus aureus in animals: a review. The Veterinary Journal, 175:27-36.

Linares, J. A. and Wigle, W. L. Staphylococcus aureus pneumonia in turkey poults with gross lesions resembling Aspergillosis. Avian Disease 45,1068-1072 (2001)

Liu W, Sun Z, Zhang J, Gao W, Wang W, Wu L, Sun T, Chen W, Liu X, Zhang H. (2009)Analysis of microbial composition in acid whey for dairy fan making in Yunnan by conventional method and 16S rRNA sequencing. Curr Microbiol.59(2):199-205.

Livermore, D.M. Antibiotic resistance in

staphylococci.Internat. J. of Antimicrobial Agents, 16, 3-10(2000)

Long KS, Poehlsgaard J, Kehrenberg C, Schwarz S, Vester B(2006). The Cfr rRNA methyltransferase confers resistance to phenicols, lincosamides, oxazolidinones, pleuromutilins, and streptogramin A antibiotics. Antimicrob. Agents Chemother., 50: 2500-2505.

Lowder BV, Guinane CM, Zakour NLB, Weinert LA, Conway-Morris A, Cartwright RA, Simpson AJ, Rambaut A, Nübel U, Fitzgerald JR(2009) . Recent human-to-poultry host jump, adaptation, and pandemic spread of Staphylococcus aureus. Proceedings of the National Academy of Sciences, 106:19545-19550.

Malaviolle X, Nonhoff C, Denis O, Rottiers S, Struelens MJ(2008) . Evaluation of disc diffusion methods and Vitek 2 automated system for testing susceptibility to mupirocin in Staphylococcus aureus. Journal of antimicrobial chemotherapy, 62:1018-1023.

Mansour AS, Wagih GE-S, Morgan SD, Elhariri M, El-Shabrawy MA, Abuelnaga AS, Elgabry E(2017)

Detection of Staphylococcus aureus enterotoxigenic strains in bovine raw milk by reversed passive latex agglutination and multiplex polymerase chain reaction. Veterinary world, 10 : 843.

Marek A, Stepień-Pyśniak D, Pyzik E, Wilczyński J, Winiarczyk S(2016) . Occurrence and characterization of Staphylococcus bacteria isolated from poultry in Western Poland. Berliner und Munchener tierarztliche Wochenschrift, 129: 147152.

Martineau F, Picard FJ, Lansac N, Ménard C, Roy PH, Ouellette M, Bergeron MG(2000) . Correlation between the Resistance Genotype Determined by Multiplex PCR Assays and the Antibiotic Susceptibility Patterns of Staphylococcus aureus andStaphylococcus epidermidis. Antimicrobial agents and chemotherapy, 44: 231-238.

Martins PD, de Almeida TT, Basso AP, de Moura TM, Frazzon J, Tondo EC, Frazzon APG (2013) . Coagulase-positive Staphylococci isolated from chicken meat: pathogenic potential and vancomycin resistance. Foodborne pathogens and disease, 10: 771-776. 
McClure J-A, Conly JM, Lau V, Elsayed S, Louie T, Hutchins W, Zhang K(2006) . Novel multiplex PCR assay for detection of the Staphylococcal virulence marker Panton-Valentine leukocidin genes and simultaneous discrimination of methicillin-susceptible from-resistant Staphylococci. Journal of clinical microbiology, 44:1141-1144.

Mkize N(2016) . Molecular detection and genetic characterization of antimicrobial resistance genes in foodborne pathogens isolated from slaughtered broiler chickens in Durban.

Monday SR, Bohach GA(1999) . Use of multiplex PCR to detect classical and newly described pyrogenic toxin genes in Staphylococcal isolates. Journal of clinical microbiology, 37: 3411-3414.

Monecke S, Ruppelt A, Wendlandt S, Schwarz S, Slickers P, Ehricht R, de Jäckel SC(2013). Genotyping of Staphylococcus aureus isolates from diseased poultry. Veterinary microbiology, 162:806-812.

MoralesG, Juan J. Picazo, Elvira Baos, Francisco J. Candel, Ana Arribi, Beatriz Pela' ez, Raquel Andrade, Mar1'a-A' ngeles de la Torre,3 Jose' Fereres, and Miguel Sa' nchez-Garc1'a( 2010)Resistance to Linezolid Is Mediated by the cfr Gene in the First Report of an Outbreak of LinezolidResistant Staphylococcus aureusClinical Infectious Diseases; 50:821-825

Motamedi H, Abadi SSR, Moosavian SM, Torabi $\mathrm{M}(2015)$. The association of Panton-Valentine leukocidin and mecA genes in Methicillin-Resistant Staphylococcus aureus isolates from patients referred to Educational Hospitals in Ahvaz, Iran. Jundishapur journal of microbiology 8 .

Mulders M, Haenen A, Geenen P, Vesseur P, Poldervaart E, Bosch T, Huijsdens X, Hengeveld P, Dam-Deisz W, Graat E(2010) . Prevalence of livestock-associated MRSA in broiler flocks and risk factors for slaughterhouse personnel in The Netherlands. Epidemiology \& Infection, 138: 743755.

Nahimana I, Francioli P, Blanc D(2006) . Evaluation of three chromogenic media (MRSAID, MRSA-Select and CHROMagar MRSA) and ORSAB for surveillance cultures of methicillinresistant Staphylococcus aureus. Clinical Microbiology and Infection, 12: 1168-1174.

Nam S, Kim M-j, Park C, Park J-G, MaengPJ, Lee G-C(2013) . Detection and genotyping of vancomycin-resistant Enterococcus spp. by multiplex polymerase chain reaction in Korean aquatic environmental samples. International journal of hygiene and environmental health, 216: 421-427.
Nazia K.K.M., Durrani N.U., Kamboh A.A., Lakho S.A., Rind R., Abro S.H., Soomro N.M. Prevalence of septic arthritis caused by Staphylococcus aureus in poultry birds at Tandojam, Pakistan. $J$. Anim. Health Prod. 2015;3:73-77.

Okolie CE, Essien UC, Idoko J(2015) . Genetic and phenotypic identification of vancomycin-resistant Staphylococcus aureus isolates from retail poultry carcasses in Omu-Aran, North-Central Nigeria. British Biotechnology Journal, 6: 87-92.

Onaolapo J, Igwe J, Bolaji R, Adeshina G, Parom S(2017) . Antibiotics Susceptibility Profile of Staphylococcus aureus Isolated from Poultry Birds in Kaduna, Nigeria. J ClinMicrobiolAntimicrob, $1: 1$.

Osman, K., Badr, J., Al-Maary, K. S., Moussa, I. M., Hessain, A. M., Girah,Z. M. A., and Saad, A. (2016).Prevalence of the antibiotic resistance genesin coagulase-positive-and negative-

Staphylococcus in chicken meat retailedto consumers. Frontiers in Microbiol., 22;7:1846.

OtaluO, Junaidu K, Chukwudi OE, Jarlath UV(2011) . Multi-drug resistant coagulase positive Staphylococcus aureus from live and slaughtered chickens in Zaria, Nigeria. International Journal of Poultry Science, 10: 871-875.

Otto, M. (2013). Coagulase-negative staphylococci as reservoirs of genes facilitating MRSA infection: staphylococcal commensal species such as Staphylococcus epidermidis are being recognized as important sources of genes promoting MRSA colonization and virulence. BioEssays 35, 4-11. doi: 10.1002/bies.201200112

Pan D, Yu Z. (2014)Intestinal microbiome of poultry and its interaction with host and diet. Gut Microbes.5(1):108-119. doi:10.4161/gmic.26945

Pereira V, Lopes C, Castro A, Silva J, Gibbs P, Teixeira $\mathrm{P}(2009)$. Characterization for enterotoxin production, virulence factors, and antibiotic susceptibility of Staphylococcus aureus isolates from various foods in Portugal. Food Microbiology, 26: 278-282.

Pesavento G, Ducci B, Comodo N, Nostro AL(2007) . Antimicrobial resistance profile of Staphylococcus aureus isolated from raw meat: A research for methicillin resistant Staphylococcus aureus (MRSA). Food control, 18:196-200.

Phillips I, Casewell M, Cox T, De Groot B, Friis C, Jones R, Nightingale C, Preston R, Waddell $J(2004)$. Does the use of antibiotics in food animals pose a risk to human health? A critical review of publiseh d data. Journal of Antimicrobial Chemotherapy, 53:28-52. 
Piette A, Verschraegen G(2009) . Role of coagulase-negative Staphylococci in human disease. Veterinary microbiology, 134: 45-54.

Pyzik E, Marek A, Stępień-Pyśniak D, UrbanChmiel R, Jarosz ŁS, Jagiełło-Podębska I (2019) . Detection of antibiotic resistance and classical enterotoxin genes in coagulase-negative Staphylococci isolated from poultry in Poland. Journal of Veterinary Research 1.

Resch, M., Nagel, V., and Hertel, $C$.

(2008).Antibiotic resistance of coagulase negative staphylococci associated with food and used in starter cultures.Inter. J. food Microbiol., 127(1): 99 104

Rice LB(2012). Mechanisms of resistance and clinical relevance of resistance to $\beta$-lactams, glycopeptides, and fluoroquinolones, Mayo Clinic Proceedings. Elsevier, pp. 198-208.

Rosenstein R., Gotz F. What distinguiseh s highly pathogenic staphylococci from medium- and nonpathogenic? Curr.Top

Microbiol.Immunol. 2013;358:33-8

Rosenstein R., Gotz F. What distinguiseh s highly pathogenic staphylococci from medium- and nonpathogenic? Curr.Top

Microbiol.Immunol. 2013;358:33-89

Rossato AM, Reiter KC, d'Azevedo PA(2018) . Coexistence of virulence genes in methicillinresistant Staphylococcus aureus clinical isolates. Rev Soc Bras Med Trop, 51:361-363

Saadat S, Solhjoo K, Norooz-Nejad M-J, Kazemi $A(2014)$. vanA and vanB positive vancomycinresistant Staphylococcus aureus among clinical isolates in Shiraz, South of Iran. Oman medical journal, 29: 335.

Sasaki T, Tsubakishita S, Tanaka Y, Sakusabe A, Ohtsuka M, Hirotaki S, Kawakami T, Fukata T, Hiramatsu K(2010). Multiplex-PCR method for species identification of coagulase-positive Staphylococci. Journal of clinical microbiology, 48: 765-769.

Seidavi A, Mirhosseini SZ, Shivazad M, Chamani M, Sadeghi AA, Pourseify R(2010) . Detection and investigation of Escherichia coli in contents of duodenum, jejunum, ileum and cecum of broilers at different ages by PCR. Asia Pacific Journal of Molecular Biology and Biotechnology, 18: 321326.

Shan W, Li J, Fang Y, Wang X, Gu D, Zhang $\mathrm{R}(2016)$. Rapid identification of methicillinresistant Staphylococcus aureus (MRSA) by the vitek MS saramis system. Current microbiology, 72: 29-32.

Sibbald M, Ziebandt A, Engelmann S, Hecker M, De Jong A, Harmsen H, Raangs G, Stokroos I, Arends J, Dubois J(2006) . Mapping the pathways to Staphylococcal pathogenesis by comparative secretomics. Microbiol. Mol. Biol. Rev., 70: 755788.

Simor AE, Goodfellow J, Louie L, Louie M(2001) . Evaluation of a new medium, oxacillin resistance screening agar base, for the detection of methicillin-resistant Staphylococcus aureus from clinical specimens. Journal of clinical microbiology, 39: 3422-3422.

Smyth JA, McNamee PT(2001) . Staphylococci, streptococci and enterococci. Poultry diseases, 191199.

Son JS, Kim EB, Lee SJ, Jun SY, Yoon SJ, Kang SH, Choi YJ(2010) . Characterization of Staphylococcus aureus derived from bovine mastitis and isolation of two lytic bacteriophages. The Journal of general and applied microbiology, 56: 347-353.

Spanu T, Sanguinetti M, D'Inzeo T, Ciccaglione D, Romano L, Leone F, Mazzella P, Fadda G(2004) . Identification of methicillin-resistant isolates of Staphylococcus aureus and coagulase-negative Staphylococci responsible for bloodstream infections with the Phoenix ${ }^{\mathrm{TM}}$ system. Diagnostic microbiology and infectious disease, 48: 221-227.

Strommenger B, Kettlitz C, Weniger T, Harmsen D, Friedrich A, Witte W(2006) . Assignment of Staphylococcus isolates to groups by spa typing, SmaI macrorestriction analysis, and multilocus sequence typing. Journal of clinical microbiology, 44: 2533-2540.

StrommengerB, Braulke C, Pasemann B, Schmidt C, Witte W(2008) . Multiplex PCR for rapid detection of Staphylococcus aureus isolates suspected to represent community-acquired strains. Journal of clinical microbiology, 46: 582-587.

Sukru K, Ugur P, Tansu T, Tugba YH(2018) . Identification of the Staphylococcus Species Which Cause Cattle Mastitis Using MALDI-TOF MS.

Šušković J, Kos B, Beganović J, Leboš Pavunc A, Habjanič K, Matošić S(2010). Antimicrobial activity-the most important property of probiotic and starter lactic acid bacteria. Food Technology and Biotechnology, 48: 296-307.

Taponen S, Pyörälä S(2009) . Coagulase-negative Staphylococci as cause of bovine mastitis-Not so different from Staphylococcus aureus? Veterinary microbiology, 134: 29-36.

Tawfiq SM(2018). Prevalence of PVL gene in some methicillin-resistant Staphylococcus sp. isolated from frozen, non frozen chickens and slaughtering workers in Kirkuk and Erbil. Tikrit Journal of Pure Science, 23: 42-47.

Teramoto H, Salaheen S, Biswas D(2016). Contamination of post-harvest poultry products with multidrug resistant Staphylococcus aureus in 
Maryland-Washington DC metro area. Food Control, 65: 132-135.

Testing ECoAS, Testing ECoAS(2016). Breakpoint tables for interpretation of MICs and zone diameters. 2016. URL: www. eucast. org/clinical_breakpoints.

Thabit AG, Ahmed EH, Mohamed AI, Yassin AS, Ibrahim MA, Amin MM(2017) . Molecular Detection of Panton-Valentine Leukocidin (PVL) and Methicillin Resistance in Staphylococcus aureus pathogen. The Egyptian Journal of Medical Microbiology, 38: 1-6.

Tiemersma EW, Bronzwaer SL, Lyytikäinen O, Degener JE, Schrijnemakers P, Bruinsma N, Monen J, Witte W, Grundmann H, Participants EARSS(2004) . Methicillin-resistant Staphylococcus aureus in Europe, 1999-2002. Emerging infectious diseases, 10:1627.

Toh S-M, Xiong L, Arias CA, et al. Acquisition of a natural resistance gene renders a clinical strain of methicillin-resistant Staphylococcus aureus resistant to the synthetic antibiotic linezolid.

MolMicrobiol 2007; 64:1506-1514

Tong SY, Davis JS, Eichenberger E, Holland TL, Fowler VG(2015) . Staphylococcus aureus infections: epidemiology, pathophysiology, clinical manifestations, and management. Clinical microbiology reviews, 28: 603-661.

Van TTH, Moutafis G, Istivan T, Tran LT, Coloe $\mathrm{PJ}(2007)$. Detection of Salmonella spp. in retail raw food samples from Vietnam and characterization of their antibiotic resistance. Appl. Environ. Microbiol., 73: 6885-6890.

Vanderhaeghen W, Hermans K, Haesebrouck F, Butaye $\quad \mathrm{P}(2010) \quad$. Methicillin-resistant Staphylococcus aureus (MRSA) in food production animals. Epidemiology \& Infection, 138: 606-625. Veras JF, do Carmo LS, Tong LC, Shupp JW, Cummings C, dos Santos DA, Cerqueira MMOP, Cantini A, Nicoli JR, Jett M(2008). A study of the enterotoxigenicity of coagulase-negative and coagulase-positive Staphylococcal isolates from food poisoning outbreaks in Minas Gerais, Brazil. International Journal of Infectious Diseases, 12:410-415.

Vintov J, Aarestrup FM, Zinn CE, Olsen JE(2003) - Association between phage types and antimicrobial resistance among bovine Staphylococcus aureus from 10 countries.Veternary Microbiology, 95:133-147.

Vuong C, Otto M(2002) . Staphylococcus epidermidis infections. Microbes and infection, 4:481-489.
Waters AE, Contente-Cuomo T, Buchhagen J, Liu CM, Watson L, Pearce K, Foster JT, Bowers J, Driebe EM, Engelthaler DM(2011). Multidrugresistant Staphylococcus aureus in US meat and poultry. Clinical Infectious Diseases, 52: 1227 1230.

Wendlandt S, Seh n J, Kadlec K, Wang Y, Li B, Zhang W, Febler AT, Wu C, Schwarz S(2015). Multidrug resistance genes in Staphylococci from animals that confer resistance to critically and highly important antimicrobial agents in human medicine. Trends in Microbiology, 23: 44-54.

Whichard JM, Gay K, Stevenson JE, Joyce KJ, Cooper KL, Omondi M, Medalla F, Jacoby GA, Barrett TJ(2007) . Human Salmonella and concurrent decreased susceptibility to quinolones and extended-spectrum cephalosporins. Emerging infectious diseases, 13:1681.

Wieliczko A, Król J, Piasecki T, Mazurkiewicz M, Staroniewicz Z(2002) . Occurrence and characteristics of Staphylococci isolated from poultry. Med Weter, 58: 348-352.

Youssef, A.I. and Hamed, D.M. (2012): Methicillin Resistant Staphylococcus aureus (MRSA) associated with arthritis in broiler farms in Ismailia province, Egypt and its zoonotic potential significance. SCVMJ, XVII: 309-321

Wang, Y., He, T., Schwarz, S., Zhao, Q., Seh n, Z., Wu, C., and Seh n, J. (2013). Multidrug resistance gene cfr in methicillin-resistant coagulasenegativeStaphylococci from chickens, ducks, and pigs in China. Inter. J. ofMedical Microbiol., 303(2): 84-87.

Zhang K, Sparling J, Chow BL, Elsayed S, Hussain Z, Church DL, Gregson DB, Louie T, Conly JM(2004) . New quadriplex PCR assay for detection of methicillin and mupirocin resistance and simultaneous discrimination of Staphylococcus aureus from coagulase-negative Staphylococci. Journal of clinical microbiology, 42: 4947-4955. 